\title{
Gauge-Fermion Unification and Flavour Symmetry
}

\author{
Tianjun $\mathrm{Li}^{1}$ \\ School of Natural Science, Institute for Advanced Study, \\ Einstein Drive, Princeton, NJ 08540, USA
}

\begin{abstract}
After we study the 6 -dimensional $\mathcal{N}=(1,1)$ supersymmetry breaking and $R$ symmetry breaking on $M^{4} \times T^{2} / Z_{n}$, we construct two $\mathcal{N}=(1,1)$ supersymmetric $E_{6}$ models on $M^{4} \times T^{2} / Z_{3}$ where $E_{6}$ is broken down to $S O(10) \times U(1)_{X}$ by orbifold projection. In Model I, three families of the Standard Model fermions arise from the zero modes of bulk vector multiplet, and the $R$ symmetry $U(1)_{F}^{I} \times S U(2)_{4_{-}}$can be considered as flavour symmetry. This may explain why there are three families of fermions in the nature. In Model II, the first two families come from the zero modes of bulk vector multiplet, and the flavour symmetry is similar. In these models, the anomalies can be cancelled, and we have very good fits to the SM fermion masses and mixings. We also comment on the $\mathcal{N}=(1,1)$ supersymmetric $E_{6}$ models on $M^{4} \times T^{2} / Z_{4}$ and $M^{4} \times T^{2} / Z_{6}$, $S U(9)$ models on $M^{4} \times T^{2} / Z_{3}$, and $S U(8)$ models on $T^{2}$ orbifolds.
\end{abstract}

PACS: $11.25 . \mathrm{Mj}$; 11.10.Kk; 04.65.+e; 11.30.Pb

Keywords: Gauge-Fermion Unification; Flavour Symmetry; $T^{2}$ Orbifolds

October 31, 2018

\footnotetext{
${ }^{1}$ E-mail: tli@sns.ias.edu. Phone Number: (609) 734-8024. Fax Number: (609) 951-4489.
} 


\section{Introduction}

Supersymmetric Grand Unified Theory (SUSY GUT) is one of the most promising candidates which describe the fundamental interactions except gravity in the nature. Supersymmetry is an elegant solution to the gauge hierarchy problem, and the Grand Unified Theory gives us a simple understanding of the quantum numbers of quarks and leptons. Moreover, the success of gauge coupling unification in the Minimal Supersymmetric Standard Model strongly supports this idea. The electroweak symmetry can be broken by radiative corrections due to the large top quark Yukawa coupling, and the very tiny neutrino masses can be realized naturally by see-saw mechanism in the SUSY GUT.

Among several candidates of the GUT gauge groups such as $\mathrm{SU}(5), \mathrm{SO}(10)$, and $\mathrm{E}_{6}, \mathrm{SO}(10)$ has several particularly attractive features. $\mathrm{SO}(10)$ is the smallest semi-simple GUT group which does not require a particular particle content to cancel the gauge anomaly. And there exists the fermion unification, i.e., all the quarks and leptons including the right-handed neutrinos in each generation are unified to a single 16 dimensional spinor representation field, and then, we have the Yukawa unification. In addition, the doublet-triplet splitting can be explained by the Dimopoulos-Wilczek mechanism [1].

However, it is very difficult to understand the fermion masses and mixings in $\mathrm{SO}(10)$ model due to the fermion unification. The Yukawa matrices have hierarchical structures and they are quite different among the quark and lepton sectors. On the one hand, all the mixing angles in the quark sector are small and the masses of quarks have hierarchical structures. The magnitude of the mass hierarchy is enormous, for instance, the ratio of the up-quark mass $m_{u}$ to the top-quark mass $m_{t}$ is approximately $10^{-5}$. On the other hand, there exist the bilarge mixings in neutrino-lepton sector from solar neutrino, atmospheric neutrino and reactor neutrino experiments $[2,3,4]$. $\theta_{12}$, which is the mixing angle for solar oscillation, is large with best fit around $32.6^{\circ}$. And $\theta_{23}$, which is the mixing angle for atmospheric oscillation, is also large with best fit around $45^{\circ}[5,6]$. However, the mixing angle $\theta_{13}$ is small, $\sin ^{2} \theta_{13} \leq 0.04$ from the CHOOZ experiment [7].

How to understand the fermion masses and mixings in $S O(10)$ model is an interesting question. The Froggatt-Nielsen (FN) mechanism is an elegant scenario to generate the hierarchical structures in Yukawa matrices [8]. Various flavour symmetries have been introduced to explain the fermion masses and mixings in the supersymmetric $S O(10)$ models, for example, $U(1)$ and extra discrete flavour symmetry [9, 10], $U(2)$ flavour symmetry [11], $S U(2) \times U(1)$ flavour symmetry [12, 13], $S U(2) \times Z_{2} \times Z_{2} \times Z_{2}$ flavour symmetry [14], $S U(3) \times U(1)$ flavour symmetry [15], $S U(3)$ flavour symmetry [16], etc. And the fermion-mass hierarchy in 5-dimensional $\mathrm{SO}(10)$ models can be realized by different fermions with different 5-dimensional wavefunction profiles. Unlike the Froggatt-Nielsen mechanism, the $\mathrm{SO}(10)$ breaking effect may directly contribute to the source of the fermion mass hierarchy [17]. Moreover, if we introduce extra 10-dimensional matter multiplets and mix or flip the $d^{c}$ and doublet $L$ in the $\mathbf{1 6}$ and those in the 10-dimensional matter multiplets by the $\mathrm{SO}(10)$ 
breaking effect, we can explain the fermion masses and mixings by introducing $U(1)$ flavour symmetry $[18,19]$. This approach can be generalized to the 5-dimensional $S O(10)$ models [20], and to the 6-dimensional $S O(10)$ models where the Yukawa couplings can also be suppressed by the volume suppression factors [21]. However, this approach loses the fermion unification. By the way, the fermion masses and mixings in the $S O(10)$ models with various flavour symmetries has been reviewed in Ref. [22].

In addition, a new kind of scenario for the GUT breakings has been discussed extensively during the last several years [23, 24, 25, 26, 27, 28]. The key point is that the supersymmetric GUT models exist in 5 or higher dimensions and are broken down to the 4-dimensional $\mathcal{N}=1$ supersymmetric Standard like Models for the zero modes due to the discrete symmetries on the extra space manifolds, which become non-trivial constraints on the multiplets and gauge generators in the GUTs [26]. The attractive models have been constructed explicitly, where the supersymmetric 5dimensional and 6-dimensional GUT models are broken down to the 4-dimensional $\mathcal{N}=1$ supersymmetric $S U(3) \times S U(2) \times U(1)^{n-3}$ models, where $n$ is the rank of GUT group, through the compactification on various orbifolds. The GUT gauge symmetry breaking and doublet-triplet splitting problems have been solved neatly by the discrete symmetry projections. And the partial gauge-fermion unification and the family symmetry as a remnant of the higher-dimensional $R$ symmetry have been discussed in the 6 -dimensional $\mathcal{N}=(1,1)$ supersymmetric $S O(10)$ model on the space-time $M^{4} \times T^{2} / Z_{3}$ where $M^{4}$ is the 4-dimensional Minkowski space-time [27]. Other interesting phenomenology, like $\mu$ problem, gauge coupling unification, non-supersymmetric GUT, gauge-Higgs unification, gauge-Yukawa unification, proton decay, etc, have also been studied [23, 24, 25, 26, 27, 28].

Before we present our results, let us ask three interesting questions

(1) The maximal supersymmetry in the supersymmetric gauge theory is the 4-dimensional $\mathcal{N}=4$ supersymmetry, why is not the 4 -dimensional $\mathcal{N}=4$ supersymmetry in the nature?

(2) Why are there three families of fermions in the Standard Model (SM) [29]?

(3) If $S O(10)$ model is correct, what is the origin of the flavour symmetry which is used to explain the fermion masses and mixings?

In this paper, we first briefly review the $T^{2} / Z_{n}$ orbifolds. And then, we consider the 6 -dimensional $\mathcal{N}=(1,1)$ supersymmetric gauge theory on the spacetime $M^{4} \times T^{2}$, which corresponds to the 4-dimensional $\mathcal{N}=4$ supersymmetry, the maximal supersymmetry for gauge theory. The $R$ symmetry in this case is $S O(2)_{56} \times S U(2)_{4_{+}} \times S U(2)_{4_{-}}$, and the transformation properties of vector multiplet under this $R$ symmetry are given. In order to preserve only the 4-dimensional $\mathcal{N}=1$ supersymmetry, we consider the supersymmetry breaking and $R$ symmetry breaking on $M^{4} \times T^{2} / Z_{n}$. We find that for $T^{2} / Z_{3}$ orbifold, the $R$ symmetry is $S O(2)_{56} \times U(1)_{4_{+}} \times S U(2)_{4_{-}}$. And for $T^{2} / Z_{4}$ and $T^{2} / Z_{6}$ orbifolds, the $R$ symmetry 
is $S O(2)_{56} \times U(1)_{4_{+}} \times U(1)_{4_{-}}$. We also present the transformation properties of the vector multiplet under these $R$ symmetries. In addition, we explain how to embed the $R$ symmetry into the $S U(4)_{R} R$ symmetry, which is the maximal $R$ symmetry for the 4-dimensional $\mathcal{N}=4$ supersymmetry. This embedding gives us an elegant way to understand the supersymmetry and $R$ symmetry breakings.

In the 6 -dimensional $\mathcal{N}=(1,1)$ supersymmetric gauge theory, there is a vector multiplet, which in the 4 -dimensional $\mathcal{N}=1$ supersymmetry language corresponds to one gauge supermultiplet and three chiral multiplets in the adjoint representation. The Standard Model fermions may come from three chiral multiplets due to the orbifold gauge symmetry breaking, which may explain why there are three families of fermions in the Standard Model. And the $R$ symmetry can give us the flavour symmetry to explain the fermion masses and mixings. To be concrete, we discuss the $\mathcal{N}=(1,1)$ supersymmetric $E_{6}$ models on the space-time $M^{4} \times T^{2} / Z_{3}$ where the $E_{6}$ gauge symmetry is broken down to $S O(10) \times U(1)_{X}$ by orbifold projection. There are three $S O(10)$ spinor representations $\mathbf{1 6}$ from the zero modes of bulk vector multiplet, which can be considered as three families of fermions. We study two models in detail. In Model I, three families of the Standard Model fermions arise from the zero modes of bulk vector multiplet, and the $R$ symmetry $U(1)_{F}^{I} \times S U(2)_{4_{-}}$can be considered as flavour symmetry. Moreover, we show that the anomalies can be cancelled, and the very good fits to the Standard Model fermion (quark, lepton, and neutrino) masses and mixings can be obtained. In this model, we can understand why there are three families of the SM fermions in the nature and the origin of flavour symmetry. However, the $U(1)_{R}^{I} R$ symmetry is not the traditional $R$ symmetry in the low energy supersymmetry phenomenology and is broken by the Higgs VEVs. Because there is no unbroken $Z_{2}$ symmetry under which the ordinary particles are even while their superpartners are odd, there are no cold dark matter candidates (for example neutralino) from the superpartners. We point out that this $U(1)_{R}^{I} R$ symmetry may be the unbroken $U(1)_{R} \mathrm{R}$ symmetry in the compactification of the weakly coupled heterotic string theory on Calabi-Yau manifolds. So, it is interesting to study its low energy phenomenology. In Model II, the first two families of the Standard Model fermions arise from the zero modes of bulk vector multiplet, and the $R$ symmetry $U(1)_{F}^{I I} \times S U(2)_{4_{-}}$can be considered as flavour symmetry. We also discuss the anomaly cancellations, and the fermion masses and mixings. Similar to those in Model I, we have very good fits to the Standard Model fermion masses and mixings. Unlike that in Model $\mathrm{I}$, the $U(1)_{R}^{I I} R$ symmetry is the traditional $U(1)_{R} R$ symmetry in the low energy supersymmetry phenomenology. However, we can not explain why there are three families of fermions in the nature.

Furthermore, we comment on the $\mathcal{N}=(1,1)$ supersymmetric $E_{6}$ models on $M^{4} \times T^{2} / Z_{4}$ and $M^{4} \times T^{2} / Z_{6}$ where there are two families of the Standard Model fermions from the zero modes of bulk vector multiplet. In these models, the $U(1)_{R}^{I I I}$ $R$ symmetry is the traditional $U(1)_{R} R$ symmetry in the low energy supersymmetry phenomenology, and the $U(1)_{F}^{I I I} \times U(1)_{R F} R$ symmetry can be considered as flavour symmetry. In addition, we comment on the $\mathcal{N}=(1,1)$ supersymmetric $S U(9)$ and $S U(8)$ models on $M^{4} \times T^{2} / Z_{3}$ where there are three or two families of the SM fermions 
from the zero modes of bulk vector multiplet, and comment on the $S U(8)$ models on $M^{4} \times T^{2} / Z_{4}$ and $M^{4} \times T^{2} / Z_{6}$. The $R$ symmetry and flavour symmetry are similar to those in $E_{6}$ models on the corresponding $T^{2}$ orbifolds.

This paper is organized as follows: in Section 2, we briefly review the $T^{2}$ orbifolds, explain the 6 -dimensional $\mathcal{N}=(1,1)$ supersymmetric gauge theory, and consider the supersymmetry breaking and $R$ symmetry breaking by orbifold projection. In Section 3, we discuss the $\mathcal{N}=(1,1)$ supersymmetric $E_{6}$ models on the space-time $M^{4} \times T^{2} / Z_{3}$. We comment on the $\mathcal{N}=(1,1)$ supersymmetric $E_{6}$ models on $M^{4} \times T^{2} / Z_{4}$ and $M^{4} \times T^{2} / Z_{6}$ in Section 4 . We also comment on the $\mathcal{N}=(1,1)$ supersymmetric $S U(9)$ models on $M^{4} \times T^{2} / Z_{3}$ and $S U(8)$ models on $T^{2}$ orbifolds in Section 5. The discussions and conclusions are given in Section 6 .

\section{6-Dimensional $\mathcal{N}=(1,1)$ Supersymmetric Gauge Theory on $M^{4} \times T^{2} / Z_{n}$}

\subsection{Discrete Symmetry on $T^{2}$ and $T^{2}$ Orbifolds}

We consider the 6-dimensional space-time which can be factorized into a product of the ordinary 4-dimensional Minkowski space-time $M^{4}$, and the torus $T^{2}$ which is homeomorphic to $S^{1} \times S^{1}$. The corresponding coordinates for the space-time are $x^{\mu}$, $(\mu=0,1,2,3), x^{5}$ and $x^{6}$. The radii for the circles along $x^{5}$ direction and $x^{6}$ direction are $R_{1}$ and $R_{2}$, respectively. We define the complex coordinate

$$
z \equiv \frac{1}{2}\left(x^{5}+i x^{6}\right)
$$

In the complex coordinate, the torus $T^{2}$ can be defined by $C^{1}$ moduloed the equivalent classes:

$$
z \sim z+\pi R_{1}, z \sim z+\pi R_{2} e^{\mathrm{i} \theta}
$$

The complete discrete symmetries on the torus are $Z_{2}, Z_{3}, Z_{4}$ and $Z_{6}$ [24]. And for $Z_{3}, Z_{4}$ and $Z_{6}$ discrete symmetries, we have to choose $R_{1}=R_{2}=R$.

For $Z_{3}$ symmetry, $\theta=2 \pi / 3$. And the equivalent class $z \sim z+\pi R e^{\mathrm{i} 2 \pi / 3}$ is equivalent to the equivalent class $z \sim z+\pi R e^{\mathrm{i} \pi / 3}$. This torus admits the $Z_{3}$ symmetry generated by

$$
\gamma_{3}: z \rightarrow e^{\mathrm{i} 2 \pi / 3} z
$$

There are three fixed points: $z=0, z=\pi R e^{\mathrm{i} \pi / 6} / \sqrt{3}$, and $z=2 \pi R e^{\mathrm{i} \pi / 6} / \sqrt{3}$. The 3 -branes can be located at the fixed points where the extra particles can be put on.

For $Z_{4}$ symmetry, $\theta=\pi / 2$. The $Z_{4}$ symmetry is generated by

$$
\gamma_{4}: z \rightarrow e^{\mathrm{i} \pi / 2} z
$$


There are two $Z_{4}$ fixed points: $z=0$ and $z=\sqrt{2} \pi R e^{\mathrm{i} \pi / 4} / 2$, and two $Z_{2}$ fixed points: $z=\pi R / 2$ and $z=\pi R e^{\mathrm{i} \pi / 2} / 2$.

For $Z_{6}$ symmetry, $\theta=\pi / 3$. The $Z_{6}$ symmetry is generated by

$$
\gamma_{4}: z \rightarrow e^{\mathrm{i} \pi / 3} z
$$

There is one $Z_{6}$ fixed point $z=0$, two $Z_{3}$ fixed points: $z=\pi R e^{\mathrm{i} \pi / 6} / \sqrt{3}$ and $z=$ $2 \pi R e^{\mathrm{i} \pi / 6} / \sqrt{3}$, and three $Z_{2}$ fixed points: $z=\sqrt{3} \pi R e^{\mathrm{i} \pi / 6} / 2, z=\pi R / 2$ and $z=$ $\pi R e^{\mathrm{i} \pi / 3} / 2$.

In short, the $T^{2} / Z_{n}$ orbifolds are obtained from $T^{2}$ moduloed the discrete symmetry $Z_{n}$. The KK mode expansions have been discussed in Ref. [24].

\subsection{6-Dimensional $\mathcal{N}=(1,1)$ Supersymmetric Gauge Theory on $M^{4} \times T^{2}$}

As we know, the 6 -dimensional $\mathcal{N}=1((0,1)$ or $(1,0))$ supersymmetric gauge theory is chiral, where the gaugino (and gravitino) and the matters (hypermultiplets) have opposite chiralities, so, it has anomalies which are usually difficult to be cancelled by introducing the hypermultiplets. Therefore, we consider the 6 -dimensional $\mathcal{N}=(1,1)$ supersymmetry, where the irreducible box anomalies and reducible anomalies vanish, and there is no global anomaly.

For the 6 -dimensional $\mathcal{N}=(1,1)$ supersymmetry, there are four supercharges $\mathcal{Q}_{\mathbf{4}_{+}, A}^{(6)}$ and $\mathcal{Q}_{\mathbf{4}_{-}, B}^{(6)}$ with $A, B=1,2$, which belong to the $\boldsymbol{4}_{+}$and $\boldsymbol{4}_{-}$spinor representations of the $S O(5,1)$ with positive and negative chiralities, respectively [30,31]. The pseudo-Majorana condition is imposed on these four supercharges,

$$
\mathcal{Q}_{\mathbf{4}_{+}, A}^{(6)}=J_{A A^{\prime}}\left(\mathcal{Q}_{\mathbf{4}_{+}, A^{\prime}}^{(6)}\right)^{c}, \mathcal{Q}_{\mathbf{4}_{-}, B}^{(6)}=J_{B B^{\prime}}\left(\mathcal{Q}_{\mathbf{4}_{-}, B^{\prime}}^{(6)}\right)^{c},
$$

where $c$ is the charge conjugation, and $J$ is a symplectic invariant metric

$$
J=\left(\begin{array}{cc}
0 & -1 \\
1 & 0
\end{array}\right)
$$

Thus, only two supercharges are independent. The $R$ symmetry for the 6-dimensional $\mathcal{N}=(1,1)$ supersymmetry is $S U(2)_{\mathbf{4}_{+}} \times S U(2)_{\mathbf{4}_{-}}$. The supercharges $\mathcal{Q}_{\mathbf{4}_{+}, A}^{(6)}$ and $\mathcal{Q}_{\mathbf{4}_{-}, B}^{(6)}$ form the fundamental representations under the $R$ symmetry $S U(2)_{4_{+}}$and $S U(2)_{4_{-}}$, respectively [30, 31].

For the gamma matrices and spinors in 4-dimension and 6-dimension, we follow the convention in Ref. [31]. After the compactification on torus, each 6-dimensional supercharge is decomposed into two 4-dimensional Weyl spinors

$$
\mathcal{Q}_{\mathbf{4}_{+}, 1}^{(6)}=\left(\begin{array}{cc}
\mathcal{Q}_{\alpha, 1}^{(4)} & 0 \\
0 & \overline{\mathcal{Q}}_{(4)}^{\dot{\alpha}, 2}
\end{array}\right) \quad, \quad \mathcal{Q}_{4_{+}, 2}^{(6)}=\left(\begin{array}{cc}
\mathcal{Q}_{\alpha, 2}^{(4)} & 0 \\
0 & -\overline{\mathcal{Q}}_{(4)}^{\dot{\alpha}, 1}
\end{array}\right)
$$




$$
\mathcal{Q}_{\mathbf{4}_{-}, 1}^{(6)}=\left(\begin{array}{cc}
0 & \mathcal{Q}_{\alpha, 3}^{(4)} \\
\overline{\mathcal{Q}}_{(4)}^{\dot{\alpha}, 4} & 0
\end{array}\right) \quad, \quad \mathcal{Q}_{\mathbf{4}_{-}, 2}^{(6)}=\left(\begin{array}{cc}
0 & \mathcal{Q}_{\alpha, 4}^{(4)} \\
-\overline{\mathcal{Q}}_{(4)}^{\dot{\alpha}, 3} & 0
\end{array}\right)
$$

where $\mathcal{Q}_{\alpha, 1}^{(4)}, \mathcal{Q}_{\alpha, 2}^{(4)}, \mathcal{Q}_{\alpha, 3}^{(4)}$, and $\mathcal{Q}_{\alpha, 4}^{(4)}$ are the usual 4-dimensional supercharges that belong to the $(\mathbf{1} / \mathbf{2}, \mathbf{0})$ or $\mathbf{2}$ spinor representation of the $S O(3,1)$, and their charge conjugations $\overline{\mathcal{Q}}_{(4)}^{\dot{\alpha}, 1}, \overline{\mathcal{Q}}_{(4)}^{\dot{\alpha}, 2}, \overline{\mathcal{Q}}_{(4)}^{\dot{\alpha}, 3}$, and $\overline{\mathcal{Q}}_{(4)}^{\dot{\alpha}, 4}$ belong to the $(\mathbf{0}, \mathbf{1} / \mathbf{2})$ or $\mathbf{2}^{\prime}$ spinor representation of the $S O(3,1)$. So, we have the 4 -dimensional $\mathcal{N}=4$ supersymmetry. After the compactification, the $S O(2)_{56}$ rotation on the $x_{5}-x_{6}$ plane, which is a subgroup of the $S O(5,1)$, becomes the $R$ symmetry in the 4-dimensional supersymmetric gauge theory. Therefore, the $R$ symmetry in the 4-dimensional supersymmetric gauge theory is $S O(2)_{56} \times S U(2)_{4_{+}} \times S U(2)_{4_{-}}$, which is the subgroup of $S U(4)_{R}$, the maximal $R$ symmetry in the 4-dimensional $\mathcal{N}=4$ supersymmetric gauge theory. $\left(\mathcal{Q}_{\alpha, 1}^{(4)}, \mathcal{Q}_{\alpha, 2}^{(4)}\right)^{T}$ form a fundamental representation of $S U(2)_{4_{+}}$, and $\left(\mathcal{Q}_{\alpha, 3}^{(4)}, \mathcal{Q}_{\alpha, 4}^{(4)}\right)^{T}$ form a fundamental representation of $S U(2)_{4_{-}}$where $T$ is the transpose. Moreover, since the supercharges transform under the $S O(5,1)$ as spinor representations, they are charged under the $S O(2)_{56}$. From the 6-dimensional chiralities, we obtain that $\mathcal{Q}_{\alpha, 1}^{(4)}$ and $\mathcal{Q}_{\alpha, 2}^{(4)}$ carry the $S O(2)_{56}$ charges $+1 / 2$, and $\mathcal{Q}_{\alpha, 3}^{(4)}$ and $\mathcal{Q}_{\alpha, 4}^{(4)}$ carry charges $-1 / 2$.

In the 6-dimensional $\mathcal{N}=(1,1)$ supersymmetric gauge theory, the vector multiplet is a unique supermultiplet of the $(1,1)$ supersymmetric theory besides the gravity multiplet. It consists of a 6 -dimensional vector field $A_{\mu}(\mu=0,1,2,3), A_{5}, A_{6}$, two pseudo-Majorana-Weyl spinors with opposite chiralities $\lambda_{\mathbf{4}_{-}, A}$ and $\lambda_{\mathbf{4}_{+}, B}$ where $A, B=1,2$, and two complex scalar fields $\sigma^{2}$ and $\sigma^{3}$. All of them are in the adjoint representation under the bulk gauge group. The vector fields $A_{\mu}, A_{5}$, and $A_{6}$ are invariant under the R-symmetry $S U(2)_{\mathbf{4}_{+}} \times S U(2)_{\mathbf{4}_{-}}$. The spinors $\left(\lambda_{\mathbf{4}_{-}, 1}, \lambda_{\mathbf{4}_{-}, 2}\right)^{T}$ transform as a fundamental representation under the $S U(2)_{\mathbf{4}_{+}}$, and the spinors $\left(\lambda_{\mathbf{4}_{+}, 1}, \lambda_{\mathbf{4}_{+}, 2}\right)^{T}$ transform as a fundamental representation under the $S U(2)_{4_{-}}$. Four real components of scalar fields $\sigma^{2}$ and $\sigma^{3}$ belong to the $(\overline{\mathbf{2}}, \overline{\mathbf{2}})$ representation under R-symmetry $S U(2)_{4_{+}} \times S U(2)_{4_{-}}$, which transforms as

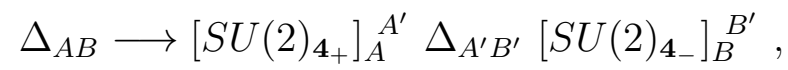

where

$$
\Delta_{A B}=\epsilon_{A^{\prime} A}\left(\begin{array}{cc}
\sigma^{2} & \sigma^{3} \\
\sigma^{3 *} & -\sigma^{2 *}
\end{array}\right)^{A^{\prime} B^{\prime}} \epsilon_{B^{\prime} B} .
$$

So, they form a vector of the $S O(4)_{\mathrm{R}} \simeq S U(2)_{4_{+}} \times S U(2)_{4_{-}}$.

If the 6 -dimensional $\mathcal{N}=(1,1)$ supersymmetric gauge theory is compactified on a torus $\mathbf{T}^{2}$, we have 4 -dimensional $\mathcal{N}=4$ supersymmetry due to the flat metric of torus. The 4 -dimensional $\mathcal{N}=4$ vector multiplet contains a 4 -dimensional vector field $A_{\mu}$, four fermion partners $\chi_{\alpha}^{a}(a=1, \ldots, 4)$, and three complex scalar fields $\sigma^{1}, \sigma^{2}, \sigma^{3}$. The four Weyl fermions $\chi_{\alpha}^{a}$ are obtained from the 6 -dimensional fermion fields $\lambda_{\boldsymbol{4}_{-}, \mathbf{A}}$ 
and $\lambda_{\mathbf{4}_{+}, \mathbf{B}}$ as the following decomposition

$$
\begin{array}{cc}
\lambda_{\mathbf{4}_{-}, 1} \rightarrow\left(\begin{array}{cc}
0 & -\chi_{\alpha}^{2} \\
\bar{\chi}_{1}^{\dot{\alpha}} & 0
\end{array}\right) \quad, \quad \lambda_{\mathbf{4}_{-}, 2} \rightarrow\left(\begin{array}{cc}
0 & \chi_{\alpha}^{1} \\
\bar{\chi}_{2}^{\dot{\alpha}} & 0
\end{array}\right), \\
\lambda_{\mathbf{4}_{+}, 1} \rightarrow\left(\begin{array}{cc}
\chi_{\alpha}^{4} & 0 \\
0 & -\bar{\chi}_{3}^{\dot{\alpha}}
\end{array}\right) \quad, \quad \lambda_{\mathbf{4}_{+}, 2} \rightarrow\left(\begin{array}{cc}
-\chi_{\alpha}^{3} & 0 \\
0 & -\bar{\chi}_{4}^{\dot{\alpha}}
\end{array}\right),
\end{array}
$$

where the $\left(\chi_{\alpha}^{1}, \chi_{\alpha}^{2}\right)^{T}$ and $\left(\chi_{\alpha}^{3}, \chi_{\alpha}^{4}\right)^{T}$ transform as the anti-fundamental representations under the $S U(2)_{4_{+}}$and $S U(2)_{4_{-}}$, respectively. And the complex field $\sigma_{1}$ is obtained from the gauge fields $A_{5}$ and $A_{6}$

$$
\sigma^{1}\left(x^{\mu}, x^{5}, x^{6}\right) \equiv \frac{1}{\sqrt{2}}\left(A_{6}\left(x^{\mu}, x^{5}, x^{6}\right)+i A_{5}\left(x^{\mu}, x^{5}, x^{6}\right)\right)
$$

which has charge -1 under the $S O(2)_{56}$ symmetry.

In short, in terms of the 4-dimensional $\mathcal{N}=1$ supersymmetry language, the 4-dimensional $\mathcal{N}=4$ vector multiplet consists of one vector multiplet $V=\left(A_{\mu}, \chi^{1}\right)$ and three chiral multiplets $\Sigma_{1}=\left(\sigma^{1}, \chi^{2}\right), \Sigma_{2}=\left(\sigma^{2}, \chi^{3}\right)$ and $\Sigma_{3}=\left(\sigma^{3}, \chi^{4}\right)$ in the adjoint representation.

\subsection{6-Dimensional $\mathcal{N}=(1,1)$ Supersymmetry Breaking on $T^{2}$ Orbifolds}

Because we can not keep the 4 -dimensional $\mathcal{N}=4$ supersymmetry at low energy from 6-dimensional $\mathcal{N}=(1,1)$ supersymmetry, we have to break it down to the 4dimensional $\mathcal{N}=1$ supersymmetry by orbifold projections from the phenomenological point of view.

Before we discuss the 6 -dimensional $\mathcal{N}=(1,1)$ supersymmetry reaking on $T^{2}$ orbifolds, we briefly review the symmetry breaking on $T^{2}$ orbifolds. Suppose $\Gamma$ is a gauge (local) or global symmetry of the Lagrangian and consider $Z_{n}(n=3,4,6)$ discrete symmetry on $T^{2}$, we have

$$
z \sim \omega z, \mathcal{L}\left(x^{\mu}, \omega z, \omega^{-1} \bar{z}\right)=\mathcal{L}\left(x^{\mu}, z, \bar{z}\right)
$$

where $\omega=e^{i 2 \pi / n}$. So, for a generic bulk multiplet $\Phi$ which fills a representation of the bulk symmetry group $\Gamma$, we have

$$
\Phi\left(x^{\mu}, \omega z, \omega^{-1} \bar{z}\right)=\eta_{\Phi}\left(R_{\omega}^{\Gamma}\right)^{l_{\Phi}} \Phi\left(x^{\mu}, z, \bar{z}\right)\left(\left(R_{\omega}^{\Gamma}\right)^{-1}\right)^{m_{\Phi}}
$$

where $\eta_{\Phi}$, which is a possible phase for the bulk multiplet $\Phi$ from Lagrangian, is an element of $Z_{n}$. And $l_{\Phi}$ and $m_{\Phi}$ are respectively the numbers of the fundamental index and anti-fundamental index for the bulk multiplet $\Phi$ under the bulk symmetry group $\Gamma$. For example, if $\Gamma$ is a $S U(N)$ group, for fundamental representation, $l_{\Phi}=1$, $m_{\Phi}=0$, and for adjoint representation, $l_{\Phi}=1, m_{\Phi}=1$. Moreover, $R_{\omega}^{\Gamma}$ is the 
representation of the $Z_{n}$ symmetry element $z \rightarrow \omega z$, which is introduced to break the bulk symmetry group $\Gamma$. Also, $R_{\omega}^{\Gamma}$ is an element in $\Gamma$, and satisfies $\left(R_{\omega}^{\Gamma}\right)^{n}=1$ due to $\omega^{n}=1$. Because we disscuss the supersymmetry breaking and $R$ symmetry breaking in this subsection, we choose $\eta_{\Phi}=1$ in the following discussions.

Let us discuss the supersymmetry and $R$ symmetry breakings. First, we consider the simple case where $R_{\omega}^{S U(2)_{4_{+}}}$and $R_{\omega}^{S U(2)_{4_{-}}}$are trivial

$$
R_{\omega}^{S U(2)_{4_{+}}}=\operatorname{diag}[+1,+1], R_{\omega}^{S U(2) 4_{-}}=\operatorname{diag}[+1,+1] .
$$

Under $Z_{n}$ symmetry

$$
\gamma_{n}: z \rightarrow \omega z
$$

i.e., $S O(2)_{56}$ rotation with angle $2 \pi / n$, we obtain that

$$
\begin{gathered}
A_{\mu}\left(x^{\mu}, \omega z, \omega^{-1} \bar{z}\right)=A_{\mu}\left(x^{\mu}, z, \bar{z}\right), \sigma^{1}\left(x^{\mu}, \omega z, \omega^{-1} \bar{z}\right)=\omega^{-1} \sigma^{1}\left(x^{\mu}, z, \bar{z}\right), \\
\sigma^{2}\left(x^{\mu}, \omega z, \omega^{-1} \bar{z}\right)=\sigma^{2}\left(x^{\mu}, z, \bar{z}\right), \sigma^{3}\left(x^{\mu}, \omega z, \omega^{-1} \bar{z}\right)=\sigma^{3}\left(x^{\mu}, z, \bar{z}\right), \\
\mathcal{Q}_{\alpha, 1}^{(4)}\left(x^{\mu}, \omega z, \omega^{-1} \bar{z}\right)=\omega^{1 / 2} \mathcal{Q}_{\alpha, 1}^{(4)}\left(x^{\mu}, z, \bar{z}\right), \\
\mathcal{Q}_{\alpha, 2}^{(4)}\left(x^{\mu}, \omega z, \omega^{-1} \bar{z}\right)=\omega^{1 / 2} \mathcal{Q}_{\alpha, 2}^{(4)}\left(x^{\mu}, z, \bar{z}\right), \\
\mathcal{Q}_{\alpha, 3}^{(4)}\left(x^{\mu}, \omega z, \omega^{-1} \bar{z}\right)=\omega^{-1 / 2} \mathcal{Q}_{\alpha, 3}^{(4)}\left(x^{\mu}, z, \bar{z}\right) \\
\mathcal{Q}_{\alpha, 4}^{(4)}\left(x^{\mu}, \omega z, \omega^{-1} \bar{z}\right)=\omega^{-1 / 2} \mathcal{Q}_{\alpha, 4}^{(4)}\left(x^{\mu}, z, \bar{z}\right) \\
\chi^{1}\left(x^{\mu}, \omega z, \omega^{-1} \bar{z}\right)=\omega^{-1 / 2} \chi^{1}\left(x^{\mu}, z, \bar{z}\right), \chi^{2}\left(x^{\mu}, \omega z, \omega^{-1} \bar{z}\right)=\omega^{-1 / 2} \chi^{2}\left(x^{\mu}, z, \bar{z}\right), \\
\chi^{3}\left(x^{\mu}, \omega z, \omega^{-1} \bar{z}\right)=\omega^{1 / 2} \chi^{3}\left(x^{\mu}, z, \bar{z}\right), \chi^{4}\left(x^{\mu}, \omega z, \omega^{-1} \bar{z}\right)=\omega^{1 / 2} \chi^{4}\left(x^{\mu}, z, \bar{z}\right) .
\end{gathered}
$$

From Eqs. (21-26), we obtain that the 4-dimensional $\mathcal{N}=4$ supersymmetry is completely broken.

In order to preserve the 4 -dimensional $\mathcal{N}=1$ supersymmetry and make sure that all the bulk fields have KK modes, we choose

$$
R_{\omega}^{S U(2)_{4_{+}}}=\operatorname{diag}\left[\omega^{-1 / 2}, \omega^{+1 / 2}\right], R_{\omega}^{S U(2)_{4_{-}}}=\operatorname{diag}\left[\omega^{3 / 2+k}, \omega^{-3 / 2-k}\right],
$$

where $k$ is an integer, and $0 \leq k<n$. Then, we have

$$
V\left(x^{\mu}, \omega z, \omega^{-1} \bar{z}\right)=V\left(x^{\mu}, z, \bar{z}\right), \Sigma_{1}\left(x^{\mu}, \omega z, \omega^{-1} \bar{z}\right)=\omega^{-1} \Sigma_{1}\left(x^{\mu}, z, \bar{z}\right),
$$




$$
\Sigma_{2}\left(x^{\mu}, \omega z, \omega^{-1} \bar{z}\right)=\omega^{-1-k} \Sigma_{2}\left(x^{\mu}, z, \bar{z}\right), \Sigma_{3}\left(x^{\mu}, \omega z, \omega^{-1} \bar{z}\right)=\omega^{2+k} \Sigma_{3}\left(x^{\mu}, z, \bar{z}\right) .
$$

From Eqs. (28) and (29), we obtain that for the zero modes, the 4-dimensional $\mathcal{N}=4$ supersymmetry is broken down to the 4-dimensional $\mathcal{N}=1$ supersymmetry if and only if $1+k \neq 0 \bmod n$ and $2+k \neq 0 \bmod n$, because in this case only the gauge multiplet $V$ has zero modes.

From Eq. (27), we obtain that for $k=0$ and $Z_{3}$ discrete symmetry $\left(T^{2} / Z_{3}\right.$ orbifold), the $R$ symmetry is $S O(2)_{56} \times U(1)_{4_{+}} \times S U(2)_{4_{-}}$because $R_{\omega}^{S U(2)_{4_{+}}}$breaks the $S U(2)_{\boldsymbol{4}_{+}}$R symmetry down to $U(1)_{\boldsymbol{4}_{+}}$, and $R_{\omega}^{S U(2)_{\boldsymbol{4}_{-}}}$does not break the $S U(2)_{\boldsymbol{4}_{-}}$ $R$ symmetry. And for the rest cases, the $R$ symmetry is $S O(2)_{56} \times U(1)_{4_{+}} \times U(1)_{4_{-}}$ because $R_{\omega}^{S U(2)_{4_{+}}}$and $R_{\omega}^{S U(2)_{4_{-}}}$breaks the $S U(2)_{4_{+}} \times S U(2)_{4_{-}} R$ symmetry down to $U(1)_{4_{+}} \times U(1)_{4_{-}}$.

In addition, to preserve the 4 -dimensional $\mathcal{N}=1$ supersymmetry, we find that for the $T^{2} / Z_{3}$ orbifold, the only possibility is $k=0$. And in this case, the $R$ symmetry is $S O(2)_{56} \times U(1)_{4_{+}} \times S U(2)_{4_{-}}$. Moreover, for the $T^{2} / Z_{4}$ orbifold, $k=0$ is equivalent to $k=1$, i.e., there is also only one possibility. For the $T^{2} / Z_{6}$ orbifold, the inequivalent cases are $k=0$ and $k=1$. The $R$ symmetry for $T^{2} / Z_{4}$ and $T^{2} / Z_{6}$ orbifolds is $S O(2)_{56} \times U(1)_{4_{+}} \times U(1)_{4_{-}}$.

The maximal $R$ symmetry for the 4-dimensional $\mathcal{N}=4$ supersymmetry is $S U(4)_{R}$. Embedding the $S O(2)_{56} \times S U(2)_{4_{+}} \times S U(2)_{4_{-}} R$ symmetry into the $S U(4)_{R}$ gives us an elegant way to describe the supersymmetry and $R$ symmetry breakings, so, we study it in detail.

The generator of $S O(2)_{56}$ in $S U(4)_{R}$ is

$$
T_{S O(2)_{56}}=\operatorname{diag}\left[+\frac{1}{2},+\frac{1}{2},-\frac{1}{2},-\frac{1}{2}\right],
$$

and the generators of $S U(2)_{4_{+}} \times S U(2)_{4_{-}}$are embedded into $S U(4)_{R}$ as

$$
\left(\begin{array}{cc}
T_{S U(2)_{4_{+}}} & 0 \\
0 & T_{S U(2)_{4_{-}}}
\end{array}\right) \subset\left(T_{S U(4)_{R}}\right) .
$$

We define

$$
\begin{gathered}
\left(\mathcal{Q}_{\alpha}^{(4)}\right)^{T} \equiv\left(\mathcal{Q}_{\alpha, 1}^{(4)}, \mathcal{Q}_{\alpha, 2}^{(4)}, \mathcal{Q}_{\alpha, 3}^{(4)}, \mathcal{Q}_{\alpha, 4}^{(4)}\right), \\
\left(\chi_{\alpha}\right)^{T} \equiv\left(\chi_{\alpha}^{1}, \chi_{\alpha}^{2}, \quad \chi_{\alpha}^{3}, \quad \chi_{\alpha}^{4}\right), \\
\sigma^{a b} \equiv\left(\begin{array}{cccc}
0 & \sigma^{1} & \sigma^{2} & \sigma^{3} \\
-\sigma^{1} & 0 & \sigma^{3 *} & -\sigma^{2 *} \\
-\sigma^{2} & -\sigma^{3 *} & 0 & \sigma^{1 *} \\
-\sigma^{3} & \sigma^{2 *} & -\sigma^{1 *} & 0
\end{array}\right) .
\end{gathered}
$$


And then, $\mathcal{Q}_{\alpha}^{(4)}$ transforms as the fundamental representation 4 under $S U(4)_{R}, \chi_{\alpha}$ transforms as the anti-fundamental representation $\overline{4}$ under $S U(4)_{R}$, and $\sigma^{a b}$ transforms as the anti-symmetric tensor $\overline{4} \wedge \overline{4}$ of two anti-fundamental representations under $S U(4)_{R}$.

Similar to the weakly-coupled heterotic string compactification, in order to preserve the 4-dimensional $\mathcal{N}=1$ supersymmetry, $R_{\omega}^{S U(4)_{R}}$ should take the following form

$$
R_{\omega}^{S U(4)_{R}}=\left(\begin{array}{cc}
1 & 0 \\
0 & R_{\omega}^{S U(3)_{R}}
\end{array}\right)
$$

The supercharges $\left(\mathcal{Q}_{\alpha, 2}^{(4)}, \mathcal{Q}_{\alpha, 3}^{(4)}, \mathcal{Q}_{\alpha, 4}^{(4)}\right)^{T}$ form a fundamental representation under the $S U(3)_{R},\left(\chi_{\alpha}^{2}, \chi_{\alpha}^{3}, \chi_{\alpha}^{4}\right)^{T}$ and $\left(\sigma^{1}, \sigma^{2}, \sigma^{3}\right)^{T}$ form the anti-fundamental representation under the $S U(3)_{R}$.

With Eqs. (27), (30) and (31), we obtain

$$
\begin{aligned}
R_{\omega}^{S U(4)_{R}}= & \operatorname{diag}\left[\omega^{+1 / 2}, \omega^{+1 / 2}, \omega^{-1 / 2}, \omega^{-1 / 2}\right] \times \operatorname{diag}\left[\omega^{-1 / 2}, \omega^{+1 / 2}, 1,1\right] \\
& \times \operatorname{diag}\left[1,1, \omega^{3 / 2+k}, \omega^{-3 / 2-k}\right] .
\end{aligned}
$$

Thus, we have

$$
R_{\omega}^{S U(4)_{R}}=\operatorname{diag}\left[+1, \omega, \omega^{1+k}, \omega^{-2-k}\right] .
$$

Similar to above discussions, for the zero modes, the 4-dimensional $\mathcal{N}=4$ supersymmetry is broken down to the 4 -dimensional $\mathcal{N}=1$ supersymmetry if and only if $1+k \neq 0 \bmod n$ and $2+k \neq 0 \bmod n$. And the transformations of vector multiplet under $Z_{n}$ are given by Eqs. (28) and (29).

Suppose $G$ is a Lie group and $H$ is a subgoup of $G$, for our convention, we denote the commutant of $H$ in $G$ as $G / H$, i.e.,

$$
G / H \equiv\{g \in G \mid g h=h g, \text { for any } h \in H\} \text {. }
$$

With this convention, the unbroken $R$ symmetry is given by

$$
\left(S U(4)_{R} / R_{\omega}^{S U(4)_{R}}\right) \cap\left(S O(2)_{56} \times S U(2)_{\mathbf{4}_{+}} \times S U(2)_{\mathbf{4}_{-}}\right) .
$$

Similar to above results, to preserve the 4-dimensional $\mathcal{N}=1$ supersymmetry, we obtain that for $T^{2} / Z_{3}$ orbifold, the only possibility is $k=0$ and the $R$ symmetry is $S O(2)_{56} \times U(1)_{4_{+}} \times S U(2)_{4_{-}}$. And for $T^{2} / Z_{4}$ orbifold, the only possibility is $k=0$, too. For the $T^{2} / Z_{6}$ orbifold, there are two possibilities: $k=0$ and $k=1$. The $R$ symmetry for $T^{2} / Z_{4}$ and $T^{2} / Z_{6}$ orbifolds is $S O(2)_{56} \times U(1)_{4_{+}} \times U(1)_{4_{-} \text {. }}$.

To be concrete, we define the generators for $U(1)_{4_{+}}$and $U(1)_{4_{-}}$in the $S U(4)_{R}$

$$
T_{U(1)_{4_{+}}} \equiv \operatorname{diag}\left[+\frac{1}{2},-\frac{1}{2}, 0,0\right]
$$




$$
T_{U(1)_{4_{-}}} \equiv \operatorname{diag}\left[0,0,+\frac{1}{2},-\frac{1}{2}\right] .
$$

To be complete, for the bulk gauge group $G$, we write down the bulk action in the Wess-Zumino gauge and 4 -dimensional $\mathcal{N}=1$ supersymmetry language [32, 33]

$$
\begin{aligned}
S= & \int d^{6} x\left\{\operatorname{Tr}\left[\int d^{2} \theta\left(\frac{1}{4 k g^{2}} \mathcal{W}^{\alpha} \mathcal{W}_{\alpha}+\frac{1}{k g^{2}}\left(\Sigma_{3} \partial \Sigma_{2}-\frac{1}{\sqrt{2}} \Sigma_{1}\left[\Sigma_{2}, \Sigma_{3}\right]\right)\right)+\text { h.c. }\right]\right. \\
& +\int d^{4} \theta \frac{1}{k g^{2}} \operatorname{Tr}\left[\left(\sqrt{2} \partial^{\dagger}+\Sigma_{1}^{\dagger}\right) e^{-V}\left(-\sqrt{2} \partial+\Sigma_{1}\right) e^{V}\right] \\
& \left.+\int d^{4} \theta \frac{1}{k g^{2}} \operatorname{Tr}\left[\Sigma_{2}^{\dagger} e^{-V} \Sigma_{2} e^{V}+\Sigma_{3}^{\dagger} e^{-V} \Sigma_{3} e^{V}\right]\right\} .
\end{aligned}
$$

From above action, we obtain the transformations of vector multiplet

$$
\begin{aligned}
& V\left(x^{\mu}, \omega z, \omega^{-1} \bar{z}\right)=\left(R_{\omega}^{G}\right)^{l_{V}} V\left(x^{\mu}, z, \bar{z}\right)\left(\left(R_{\omega}^{G}\right)^{-1}\right)^{m_{V}},
\end{aligned}
$$

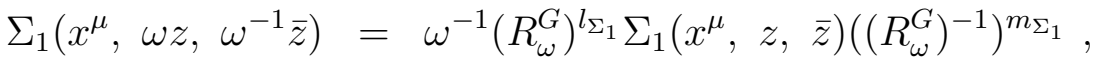

$$
\begin{aligned}
& \Sigma_{2}\left(x^{\mu}, \omega z, \omega^{-1} \bar{z}\right)=\omega^{-1-k}\left(R_{\omega}^{G}\right)^{l_{\Sigma_{2}} \Sigma_{2}}\left(x^{\mu}, z, \bar{z}\right)\left(\left(R_{\omega}^{G}\right)^{-1}\right)^{m_{\Sigma_{2}}},
\end{aligned}
$$

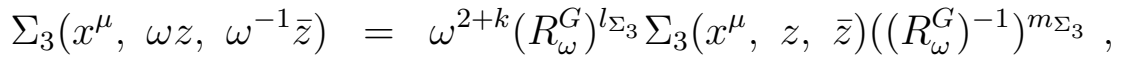

where we introduce the non-trivial $R_{\omega}^{G}$ to break the bulk gauge group $G$. When we consider the gauge symmetry breaking by discrete symmetry on the extra space manifold, we can decompose the gauge fields of $G$ under its maximal subgroup, and then, discuss the gauge symmetry breaking, for example, the $E_{6}$ breaking and $E_{8}$ breaking in Refs. [25] and [26], respectively. Some of the gauge fields of $G$ are not in the adjoint representations under its maximal subgroup due to decomposition, so, we write down the explicit $l_{\Phi}$ and $m_{\Phi}$ for the general orbifold gauge symmetry breaking.

Moreover, under the $S O(2)_{56} R$ symmetry, the $\theta$ (the Grassmann coordinate for the unbroken 4-dimensional $\mathcal{N}=1$ supersymmetry), $z$ and the bulk vector multiplet transform as

$$
\begin{gathered}
z \longrightarrow e^{\mathrm{i} \beta} z, \theta \longrightarrow e^{-\mathrm{i} \beta / 2} \theta, V \longrightarrow V, \\
\Sigma_{1} \longrightarrow e^{-\mathrm{i} \beta} \Sigma_{1}, \Sigma_{2} \longrightarrow \Sigma_{2}, \Sigma_{3} \longrightarrow \Sigma_{3} .
\end{gathered}
$$

Under the $U(1)_{4_{+}} R$ symmetry, the Grassmann coordinate $\theta, z$ and the bulk vector multiplet transform as

$$
\begin{gathered}
z \longrightarrow z, \theta \longrightarrow e^{-\mathrm{i} \beta / 2} \theta, V \longrightarrow V, \\
\Sigma_{1} \longrightarrow \Sigma_{1}, \Sigma_{2} \longrightarrow e^{-\mathrm{i} \beta / 2} \Sigma_{2}, \Sigma_{3} \longrightarrow e^{-\mathrm{i} \beta / 2} \Sigma_{3} .
\end{gathered}
$$

For the $T^{2} / Z_{3}$ orbifold, $\left(\Sigma_{2}, \Sigma_{3}\right)^{T}$ form an anti-fundamental representation under $S U(2)_{4_{-}}$. And for the $T^{2} / Z_{4}$ and $T^{2} / Z_{6}$ orbifolds, $\Sigma_{2}$ and $\Sigma_{3}$ have the $U(1)_{4_{-}}$ charges $-1 / 2$ and $+1 / 2$, respectively, while $\theta, z, V$ and $\Sigma_{1}$ are neutral. 


\section{$3 \mathcal{N}=(1,1)$ Supersymmetric $E_{6}$ Models on $M^{4} \times$ $T^{2} / Z_{3}$}

In this Section, we discuss the $\mathcal{N}=(1,1)$ supersymmetric $E_{6}$ models on the spacetime $M^{4} \times T^{2} / Z_{3}$ where the gauge symmetry $E_{6}$ is broken down to $S O(10) \times U(1)_{X}$ by orbifold projection. And there are three $S O(10)$ spinor representation $\mathbf{1 6}$ s from the zero modes of bulk vector multiplet, which can be considered as three families of fermions. We will discuss two models in detail, and show that the fermion masses and mixings can be explained by the flavour symmetry $S U(2) \times U(1)$ whose origin is $R$ symmetry. By the way, in this Section, we consider the $T^{2} / Z_{3}$ orbifold, so, $\omega=e^{\mathrm{i} 2 \pi / 3}$.

The gauge fields of $E_{6}$ are in the adjoint representation with dimension 78 . Under the gauge group $S O(10) \times U(1)_{X}$, the $E_{6}$ gauge fields decompose as [34]

$$
78=(45,0) \oplus(\mathbf{1 6},-3) \oplus(\overline{\mathbf{1 6}}, \mathbf{3}) \oplus(\mathbf{1}, \mathbf{0}) .
$$

The $R_{\omega}^{E_{6}}$ for $E_{6}$ gauge group is the product of the $R_{\omega}^{S O(10)}$ for $S O(10)$ and $R_{\omega}^{U(1)_{X}}$ for $U(1)_{X}$

$$
R_{\omega}^{E_{6}}=R_{\omega}^{S O(10)} \otimes R_{\omega}^{U(1)_{X}}
$$

In order to break the $E_{6}$ gauge symmetry, we choose

$$
\begin{gathered}
R_{\omega}^{S O(10)=} \operatorname{diag}[+1,+1,+1,+1,+1,+1,+1,+1,+1,+1], \\
R_{\omega}^{U(1)_{X}}=\exp (-\mathrm{i} 2 \pi Q / 9),
\end{gathered}
$$

where $Q$ is the $U(1)_{X}$ charge of the field. And the sufficient condition for $R_{\omega}^{U(1)_{X}}$ to satisfy is that $\left(R_{\omega}^{U(1)_{X}}\right)^{3}$ is equal to 1 for all the bulk fields which are charged under $U(1)_{X}$.

Using Eqs. (43-46) with $k=0$ and Eqs. (52-54), we obtain the transformations of vector multiplet

$$
\begin{aligned}
V_{(\mathbf{4 5}, \mathbf{0})}\left(x^{\mu}, \omega z, \omega^{-1} \bar{z}\right) & =V_{(\mathbf{4 5}, \mathbf{0})}\left(x^{\mu}, z, \bar{z}\right) \\
V_{(\mathbf{1}, \mathbf{0})}\left(x^{\mu}, \omega z, \omega^{-1} \bar{z}\right) & =V_{(\mathbf{1}, \mathbf{0})}\left(x^{\mu}, z, \bar{z}\right) \\
V_{(\mathbf{1 6},-\mathbf{3})}\left(x^{\mu}, \omega z, \omega^{-1} \bar{z}\right) & =\omega V_{(\mathbf{1 6},-\mathbf{3})}\left(x^{\mu}, z, \bar{z}\right) \\
V_{(\overline{\mathbf{1 6}}, \mathbf{3})}\left(x^{\mu}, \omega z, \omega^{-1} \bar{z}\right) & =\omega^{2} V_{(\overline{\mathbf{1 6}}, \mathbf{3})}\left(x^{\mu}, z, \bar{z}\right), \\
\left(\Sigma_{i}\right)_{(\mathbf{4 5 , 0})}\left(x^{\mu}, \omega z, \omega^{-1} \bar{z}\right) & =\omega^{2}\left(\Sigma_{i}\right)_{(\mathbf{4 5}, \mathbf{0})}\left(x^{\mu}, z, \bar{z}\right),
\end{aligned}
$$




$$
\begin{aligned}
& \left(\sum_{i}\right)_{(\mathbf{1}, \mathbf{0})}\left(x^{\mu}, \omega z, \omega^{-1} \bar{z}\right)=\omega^{2}\left(\Sigma_{i}\right)_{(\mathbf{1}, \mathbf{0})}\left(x^{\mu}, z, \bar{z}\right) \\
& \left(\Sigma_{i}\right)_{(\mathbf{1 6},-\mathbf{3})}\left(x^{\mu}, \omega z, \omega^{-1} \bar{z}\right)=\left(\Sigma_{i}\right)_{(\mathbf{1 6},-\mathbf{3})}\left(x^{\mu}, z, \bar{z}\right) \\
& \left(\Sigma_{i}\right)_{(\overline{\mathbf{1 6}}, \mathbf{3})}\left(x^{\mu}, \omega z, \omega^{-1} \bar{z}\right)=\omega\left(\sum_{i}\right)_{(\overline{\mathbf{1 6}}, \mathbf{3})}\left(x^{\mu}, z, \bar{z}\right)
\end{aligned}
$$

where $i=1,2,3$, and $\omega=e^{\mathrm{i} 2 \pi / 3}$.

From the general discussions on gauge symmetry breaking by discrete symmetry [26], we obtain that only the fields $V_{(\mathbf{4 5 , 0})}, V_{(\mathbf{1 , 0})}$, and $\left(\Sigma_{i}\right)_{(\mathbf{1 6},-\mathbf{3})}$ have zero modes. So, the $E_{6}$ gauge symmetry is broken down to $S O(10) \times U(1)_{X}$ by orbifold projection for the zero modes. And there are three $S O(10)$ spinor representations $\mathbf{1 6}$ from the zero modes of bulk vector multiplet, which can be considered as three families of fermions. This may explain why there are three families of fermions in the nature. Moreover, the $R$ symmetry is $S O(2)_{56} \times U(1)_{4_{+}} \times S U(2)_{4_{-}}$, which can be the origin of flavour symmetry to generate the fermion masses and mixings.

\subsection{Model I}

In model I, the zero modes $\left(\Sigma_{i}\right)_{(\mathbf{1 6},-\mathbf{3})}^{(0)}$ of $\left(\Sigma_{i}\right)_{(\mathbf{1 6},-\mathbf{3})}$ where $i=1,2,3$ are considered as three families of fermions, and the $S O(2)_{56} \times U(1)_{4_{+}} \times S U(2)_{4_{-}} R$ symmetry can give us the flavour symmetry to generate the fermion masses and mixings.

To be convenient in discussions, we define

$$
16_{1} \equiv-\left(\Sigma_{3}\right)_{(16,-3)}^{(0)}, 16_{2} \equiv\left(\Sigma_{2}\right)_{(16,-3)}^{(0)}, 16_{3} \equiv\left(\Sigma_{1}\right)_{(16,-3)}^{(0)},
$$

where $\mathbf{1 6}_{i}$ is the $i$ - th family of the Standard Model fermions. In this convention, the first two families $\left(\mathbf{1 6}_{1}, \mathbf{1 6}_{2}\right)^{T}$ form a fundamental representation under the $R$ symmetry $S U(2)_{4_{-}}$.

In order to give the same $U(1)_{R}$ charges for three families of fermions $\mathbf{1 6}_{i}$, we define the $U(1)_{R}^{I}$ and $U(1)_{F}^{I}$ global symmetries from the $S O(2)_{56} \times U(1)_{4_{+}} R$ symmetry, whose generators in $S U(4)_{R}$ are

$$
\begin{gathered}
T_{U(1)_{R}^{I}}=\operatorname{diag}\left[+\frac{3}{2},-\frac{1}{2},-\frac{1}{2},-\frac{1}{2}\right], \\
T_{U(1)_{F}^{I}}=\operatorname{diag}\left[0,-1,+\frac{1}{2},+\frac{1}{2}\right] .
\end{gathered}
$$

So, under $U(1)_{R}^{I}$, the Grassmann coordinate $\theta$ has charge $-3 / 2$, and three families of fermions (superfields) $\mathbf{1 6}_{i}$ have the same charge -1 . Then, the superpotential has $U(1)_{R}^{I}$ charge -3 . In addition, under $U(1)_{F}^{I}$, the Grassmann coordinate $\theta$ has charge $0, \mathbf{1 6}_{3}$ has charge +1 , and $\mathbf{1 6}_{1}$ and $\mathbf{1 6}_{2}$ have charge $-1 / 2$. 
In Model I, the $R$ symmetry $U(1)_{F}^{I} \times S U(2)_{4_{-}}$can be considered as flavour symmetry. People have discussed the fermion masses and mixings extensively in the supersymmetric $S O(10)$ models with $U(2)$ or $S U(2) \times U(1)^{n}$ flavour symmetries [11, $12,13,14]$. In this paper, we follow the discussions in Ref. [12], where the flavour symmetry is $S U(2) \times U(1)$ and the fermion masses and mixings can be explained.

To simplify the convention, we denote $\mathbf{1 6}_{1}$ and $\mathbf{1 6}_{2}$ as $\mathbf{1 6}_{a^{\prime}}$ where the low index $a^{\prime}$ is the $S U(2)_{4_{-}}$index for fundamental representation. And the upper index $a^{\prime}$ is the $S U(2)_{4_{-}}$index for anti-fundamental representation. We also assume that the particles, which are introduced to break the gauge symmetry and generate the fermion masses and mixings, are on the observable 3-brane at the fixed point $z=0$. Similar to the particle content in Ref. [12], we introduce a $\mathbf{1 0}$ dimensional Higgs field $H$, a 45 dimensional Higgs field $45_{H}$ which has a VEV along the $B-L$ direction, and one pair of $\mathbf{1 6}$ and $\overline{\mathbf{1 6}}$ Higgs fields $\left(\psi_{H}, \bar{\psi}_{H}\right)$ which are assumed to have the VEVs along the "right-handed" neutrino direction and break the $S O(10)$ gauge symmetry down to $S U(5) . \bar{\psi}_{H}$ can generate the right-handed neutrino Majorana masses. Moreover, we introduce three pairs of heavy $\mathbf{1 6}$ s and $\overline{\mathbf{1 6}}$ s of $S O(10):(\psi, \bar{\psi}),\left(\psi^{a^{\prime}}, \bar{\psi}_{a^{\prime}}\right),\left(\psi_{a^{\prime}}\right.$, $\left.\bar{\psi}^{a^{\prime}}\right)$. These heavy $16 \mathrm{~s}$ and $\overline{\mathbf{1 6}} \mathrm{s}$ do not have VEVs, and we can obtain the nonrenormalizable Yukawa matrices after we integrate them out. We also add three $S O(10)$ singlets $N_{a^{\prime}}$ and $N_{3}$.

In order to break the flavour symmetry $U(1)_{F}^{I} \times S U(2)_{4_{-}}$, we introduce an antifundamental representation $\phi^{a^{\prime}}$ (doublet), a symmetric representation with two antifundamental indices $S^{a^{\prime} b^{\prime}}$ (triplet), and an anti-symmetric representation with two anti-fundamental indices $A^{a^{\prime} b^{\prime}}$ (singlet) where the representations are under $S U(2)_{4_{-}}$ symmetry. In addition, $\phi^{a^{\prime}}, S^{a^{\prime} b^{\prime}}$, and $A^{a^{\prime} b^{\prime}}$ are $S O(10)$ singlets and charged under $U(1)_{F}^{I}$.

The quantum numbers for above particles in Model I under $U(1)_{X}$ gauge symmetry and $U(1)_{R}^{I} \times U(1)_{F}^{I} R$ symmetry are given in Table 1 where $Q_{X}, Q_{R}$ and $Q_{F}$ are free parameters.

\section{Low Energy $R$ Symmetry.}

As we know, under the traditional $U(1)_{R}$ symmetry defined in the low energy supersymmetry phenomenology, the Grassmann coordinate $\theta$ and the superfields $\mathbf{1 6}_{i}$ have charge +1 , while the Higgs fields $\left(H\right.$ and $\left.\mathbf{4 5}_{H}\right)$ have charge zero. So, the $U(1)_{R}^{I}$ symmetry defined in Eq. (64) is not the traditional $U(1)_{R}$ symmetry, and it is broken by the VEVs of $H, \psi_{H}$ and $\bar{\psi}_{H}$ (The VEVs of $\phi^{a^{\prime}}, S^{a^{\prime} b^{\prime}}$, and $A^{a^{\prime} b^{\prime}}$ will not break the $U(1)_{R}^{I}$ symmetry if $Q_{R}=0$.). Moreover, there is no unbroken $Z_{2}$ symmetry under which the ordinary particles are even while their superpartners are odd. Thus, there are no cold dark matter candidates (for example neutralino) from the superpartners, although the axion can still be the cold dark matter candidate. However, this $U(1)_{R}^{I}$ symmetry can still forbid the dimension- 5 proton decay operators $\mathbf{1 6}_{i} \mathbf{1 6}_{j} \mathbf{1 6}_{k} \mathbf{1 6}_{l}$. And we would like to point out that this $U(1)_{R}^{I}$ symmetry may be the unbroken $U(1) \mathrm{R}$ symmetry in the compactification of the weakly coupled heterotic string theory on 
Calabi-Yau manifolds. So, it is interesting to study its low energy phenomenology. In the next subsection, we will discuss a model where the first two families come from the zero modes of bulk vector multiplet and the $U(1) R$ symmetry is the traditional $U(1)_{R} R$ symmetry at low energy supersymmetry phenomenology.

\section{Anomaly Cancellations.}

In our model, there is no $[S O(10)]^{3}$ anomaly. But, there exist the mixed anomalies $[S O(10)]^{2} U(1)_{X},\left[U(1)_{X}\right]^{3}$, and [Gravity $]^{2} U(1)_{X}$, which are localized on three 3-branes at three fixed points. All three anomalies can be cancelled by a generalized Green-Schwarz mechanism [35], and then, $U(1)_{X}$ becomes a global symmetry which should be preserved by the superpotential localized on the 3-branes at the fixed points. However, the Fayet-Iliopoulos D-terms may be generated radiatively on three 3-branes at three fixed points [36]. To avoid the supersymmetry breaking from these Fayet-Iliopoulos D-terms, we require the $U(1)_{X}$ D-flatnesses on three 3-branes at three fixed points. And to achieve the $U(1)_{X}$ D-flatnesses, we introduce one pair of the $S O(10)$ singlets with $U(1)_{X}$ charges \pm 1 on each 3 -brane, and give them non-zero VEVs. Therefore, if we assume that the anomalies are cancelled by a generalized Green-Schwarz mechanism, we do not need to consider the $U(1)_{X}$ anomalies and its breaking, although we require that the superpotential localized on the 3-branes at the fixed points preserve the $U(1)_{X}$ symmetry.

In addition, we can cancel the anomalies by introducing the vector-like particles on the 3-branes at the fixed points. The anomalies due to the bulk vector multiplets arise from the zero modes of $\left(\Sigma_{i}\right)_{(\mathbf{1 6},-\mathbf{3})}$, i.e., three families of fermions $\mathbf{1 6}_{a^{\prime}}$ and $\mathbf{1 6}_{3}$. And these anomalies distribute equally on three 3-branes at three fixed points since these fixed points are equivalent from geometry. Therefore, the anomalies on the 3-brane at each fixed point due to the bulk vector multiplet are equivalent to the anomalies from a multiplet with quantum number $(\mathbf{1 6},-\mathbf{3})$ under the $S O(10) \times U(1)_{X}$ gauge symmetry. And we just need to cancel the anomalies on the 3-brane at each fixed point.

To simplify the discussions, we choose $Q_{X}=0$. First, on the observable 3brane at $z=0$, the anomalies due to the bulk vector multiplet are cancelled by the Higgs field $\bar{\psi}_{H}$. Then, we only need to cancel the anomalies generated by the Higgs field $H$. So, we introduce one pair of 10 -plets $T_{10}$ and $T_{10}^{\prime}$ whose quantum numbers are $(\mathbf{1 0}, \mathbf{- 6})$ and $(\mathbf{1 0}, \mathbf{0})$ respectively under the $S O(10) \times U(1)_{X}$ gauge symmetry. In order to give the masses to $T_{10}$ and $T_{10}^{\prime}$, and keep the $U(1)_{X}$ D-flatness and anomaly free on the 3-brane at $z=0$, we introduce the $S O(10)$ singlets $s_{1}$ and $s_{1}^{\prime}$ with $U(1)_{X}$ charges +6 and -6 , respectively. In order to forbid the mixings between the Higgs $H$ and $T_{10}$ or between $H$ and $T_{10}^{\prime}$, we assign the following $U(1)_{R}^{I} \times U(1)_{F}^{I}$ charges for these vector-like particles: the $U(1)_{R}^{I}$ charges for $T_{10}, T_{10}^{\prime}, s_{1}$ and $s_{1}^{\prime}$ are $0,0,-3$ and 0 , respectively, and the $U(1)_{F}^{I}$ charges for $T_{10}, T_{10}^{\prime}, s_{1}$ and $s_{1}^{\prime}$ are $-1,-1,2$ and -1 , respectively. One can check that the anomalies $[S O(10)]^{2} U(1)_{X},\left[U(1)_{X}\right]^{3}$, and $[\text { Gravity }]^{2} U(1)_{X}$ are cancelled on the 3 -brane at $z=0$. And the masses of $T_{10}$ and $T_{10}^{\prime}$ can be generated by the superpotential $s_{1} T_{10} T_{10}^{\prime}$. 
Second, on the 3 -brane at $z=\pi R e^{\mathrm{i} \pi / 6} / \sqrt{3}$, we need to cancel the anomalies generated by a multiplet with quantum number $(\mathbf{1 6}, \mathbf{- 3})$ under the $S O(10) \times U(1)_{X}$ gauge symmetry. So, we introduce a pair of the $\Upsilon_{2}$ and $\Upsilon_{2}^{\prime}$ whose quantum numbers are $(\mathbf{1 6}, \mathbf{0})$ and $(\overline{\mathbf{1 6}},+\mathbf{3})$ respectively under the $S O(10) \times U(1)_{X}$ gauge symmetry. And in order to give the masses to $\Upsilon_{2}$ and $\Upsilon_{2}^{\prime}$, and keep the $U(1)_{X}$ D-flatness and anomaly free on the 3 -brane at $z=\pi R e^{\mathrm{i} \pi / 6} / \sqrt{3}$, we introduce the $S O(10)$ singlets $s_{2}$ and $s_{2}^{\prime}$ with $U(1)_{X}$ charges -3 and +3 , respectively. To forbid the mixings between the SM fermions $16_{i}$ and $\Upsilon_{2}$ and the mass terms between $\mathbf{1 6}_{i}$ and $\Upsilon_{2}^{\prime}$, we assign the following $U(1)_{R}^{I} \times U(1)_{F}^{I}$ charges for the vector-like particles: the $U(1)_{R}^{I}$ charges for $\Upsilon_{2}, \Upsilon_{2}^{\prime}$, $s_{2}$ and $s_{2}^{\prime}$ are $0,0,-3$ and 0 , respectively, and the $U(1)_{F}^{I}$ charges for $\Upsilon_{2}, \Upsilon_{2}^{\prime}, s_{2}$ and $s_{2}^{\prime}$ are $-1 / 2,-1 / 2,1$ and -1 , respectively. In short, the anomalies $[S O(10)]^{2} U(1)_{X}$, $\left[U(1)_{X}\right]^{3}$, and [Gravity $]^{2} U(1)_{X}$ are cancelled on the 3 -brane at $z=\pi R e^{\mathrm{i} \pi / 6} / \sqrt{3}$. And the masses of $\Upsilon_{2}$ and $\Upsilon_{2}^{\prime}$ can be generated by the superpotential $s_{2} \Upsilon_{2} \Upsilon_{2}^{\prime}$.

Third, the anomaly cancellations on the 3-brane at $z=2 \pi R e^{\mathrm{i} \pi / 6} / \sqrt{3}$ is similar to those on the 3 -brane at $z=\pi R e^{\mathrm{i} \pi / 6} / \sqrt{3}$. We introduce the particles $\Upsilon_{3}, \Upsilon_{3}^{\prime}, s_{3}$ and $s_{3}^{\prime}$, whose quantum numbers are the same as those for $\Upsilon_{2}, \Upsilon_{2}^{\prime}, s_{2}$ and $s_{2}^{\prime}$, respectively.

Furthermore, similar to the 5-dimensional orbifold theories [37] and string theory [38], it seems to us that there exist the anomaly inflows among the 3-branes at the fixed points. If this is true, like the 5-dimensional orbifold theories, the 4-dimensional anomaly cancellations are sufficient to ensure the consistency of higher dimensional orbifold theories [37]. In other words, we only need to consider the total anomalies due to the bulk zero modes and the particles localized on the 3-branes at the fixed points. For example, we can put $\Upsilon_{2}, \Upsilon_{2}^{\prime}, s_{2}, s_{2}^{\prime}, \Upsilon_{3}$ and $\Upsilon_{3}^{\prime}$ on the 3 -brane at $z=\pi R e^{\mathrm{i} \pi / 6} / \sqrt{3}$. The anomalies are cancelled due to anomaly inflows, and $\Upsilon_{3}$ and $\Upsilon_{3}^{\prime}$ can obtain the masses via the superpotential $s_{2} \Upsilon_{3} \Upsilon_{3}^{\prime}$. The explicit proof of the anomaly inflows among the 3-branes at the fixed points on $T^{2}$ orbifolds is an interesting open question.

\section{Fermion Masses and Mixings.}

The general VEVs for $\phi^{a^{\prime}}, S^{a^{\prime} b^{\prime}}$, and $A^{a^{\prime} b^{\prime}}$, which break the $U(1)_{F}^{I} \times S U(2)_{4}$ flavour symmetry, are

$$
\begin{gathered}
\left\langle\phi^{2}\right\rangle=r\left\langle S^{22}\right\rangle,\left\langle A^{12}\right\rangle \neq 0 \\
\left\langle S^{22}\right\rangle \neq 0,\left\langle S^{11}\right\rangle=\kappa_{1}\left\langle S^{22}\right\rangle,\left\langle S^{12}\right\rangle=\kappa_{2}\left\langle S^{22}\right\rangle,
\end{gathered}
$$

where the constants $r, \kappa_{1}$ and $\kappa_{2}$ are arbitrary.

The superpotential is

$$
\begin{aligned}
W= & 16_{3} H 16_{3}+16_{a^{\prime}} H \psi^{a^{\prime}}+\bar{\psi}\left(M^{\prime} \psi+45_{H} 16_{3}\right) \\
& +\bar{\psi}_{a^{\prime}}\left(M \psi^{a^{\prime}}+\phi^{a^{\prime}} \psi+S^{a^{\prime} b^{\prime}} \psi_{b^{\prime}}+A^{a^{\prime} b^{\prime}} 16_{b^{\prime}}\right) \\
& +\bar{\psi}^{a^{\prime}}\left(M^{\prime \prime} \psi_{a^{\prime}}+45_{H} 16_{a^{\prime}}\right)+\bar{\psi}_{H}\left(N_{a^{\prime}} \psi^{a^{\prime}}+N_{3} 16_{3}\right) \\
& +\frac{1}{2} N_{a^{\prime}} N_{b^{\prime}} S^{a^{\prime} b^{\prime}}+N_{a^{\prime}} N_{3} \phi^{a^{\prime}},
\end{aligned}
$$


where

$$
\begin{gathered}
M=M_{0}\left(1+\alpha_{X^{\prime}} T_{X^{\prime}}+\beta_{Y} T_{Y}\right), \\
\alpha_{X^{\prime}} \propto \frac{\left\langle\mathbf{4 5}_{X^{\prime}}\right\rangle}{M_{0}}, \beta_{Y} \propto \frac{\left\langle\mathbf{4} \boldsymbol{5}_{Y}\right\rangle}{M_{0}},
\end{gathered}
$$

where $T_{X^{\prime}}$ and $T_{Y}$ are the generators for $U(1)_{X^{\prime}}$ and $U(1)_{Y}$, respectively where the $U(1)_{X^{\prime}}$ is the $U(1)$ in $S O(10)$ which commutes with $S U(5)$, and the $U(1)_{Y}$ is the standard weak hypercharge interaction. The Higgs fields $\mathbf{4 5} \mathbf{X}_{X^{\prime}}$ and $\mathbf{4 5} \mathbf{5}_{Y}$ have VEVs along the $U(1)_{X^{\prime}}$ and $U(1)_{Y}$ directions, respectively. And $\alpha_{X^{\prime}}$ and $\beta_{Y}$ are arbitrary constants which are used to fit the data. Futhermore, each term in superpotential has an order $1(\mathcal{O}(1))$ coupling constant which is omitted for notational simplicity. And the effective mass parameters $M_{0}, M^{\prime}$, and $M^{\prime \prime}$ can be generated by the VEVs of the $S O(10)$ singlet chiral superfields.

The mass scales are assumed to satisfy $M_{0} \sim M^{\prime} \sim M^{\prime \prime} \gg\left\langle\phi^{2}\right\rangle \sim\left\langle S^{22}\right\rangle \gg$ $\left\langle A^{12}\right\rangle$ where $M_{0}$ may be at the order of GUT scale. And the small parameters in Yukawa matrices, which are used to explain the fermion masses and mixings, are $\epsilon \sim\left\langle S^{22}\right\rangle\langle 45\rangle / M_{0}^{2}$ and $\epsilon^{\prime} \sim\left\langle A^{12}\right\rangle / M_{0}$. In this model, the second generation masses are of order $\epsilon$, while the first generation masses are of order $\epsilon^{\prime 2} / \epsilon$.

In the effective theory below $M_{0}$, after we integrate out the Froggatt-Nielsen states $\left\{\psi, \bar{\psi}, \psi^{a^{\prime}}, \bar{\psi}_{a^{\prime}}, \psi_{a^{\prime}}, \bar{\psi}^{a^{\prime}}\right\}$, we obtain the effective Yukawa matrices for uptype quarks, down-type quarks, and charged leptons, and the Dirac neutrino Yukawa matrix

$$
\begin{gathered}
Y_{u}=\left(\begin{array}{ccc}
\kappa_{1} \epsilon \rho & \left(\epsilon^{\prime}+\kappa_{2} \epsilon\right) \rho & 0 \\
-\left(\epsilon^{\prime}-\kappa_{2} \epsilon\right) \rho & \epsilon \rho & \epsilon r T_{\bar{u}} \\
0 & \epsilon r T_{Q} & 1
\end{array}\right) y, \\
Y_{d}=\left(\begin{array}{ccc}
\kappa_{1} \epsilon & \epsilon^{\prime}+\kappa_{2} \epsilon & 0 \\
-\left(\epsilon^{\prime}-\kappa_{2} \epsilon\right) & \epsilon & \epsilon r \delta T_{\bar{d}} \\
0 & \epsilon r T_{Q} & 1
\end{array}\right) y, \\
Y_{e}=\left(\begin{array}{ccc}
3 \kappa_{1} \epsilon & -\left(\epsilon^{\prime}-3 \kappa_{2} \epsilon\right) & 0 \\
\epsilon^{\prime}+3 \kappa_{2} \epsilon & 3 \epsilon & \epsilon r T_{\bar{e}} \\
0 & \epsilon r \delta T_{L} & 1
\end{array}\right) y \\
Y_{\nu}=\left(\begin{array}{ccc}
3 \kappa_{1} \epsilon \delta^{\prime} & -\left(\epsilon^{\prime}-3 \kappa_{2} \epsilon\right) \delta^{\prime} & 0 \\
\left(\epsilon^{\prime}+3 \kappa_{2} \epsilon\right) \delta^{\prime} & 3 \epsilon \delta^{\prime} & \frac{1}{2} \epsilon r \delta^{\prime} T_{\bar{\nu}} \\
0 & \epsilon r \delta T_{L} & 1
\end{array}\right) y
\end{gathered}
$$

where

$$
\rho=-\frac{5 \beta_{Y}\left(1-3 \alpha_{X^{\prime}}\right)}{16 \alpha_{X^{\prime}}\left(1+\alpha_{X^{\prime}}\right)}, \delta=\frac{1+\alpha_{X^{\prime}}}{1-3 \alpha_{X^{\prime}}}, \delta^{\prime}=\frac{2 \delta}{2 \delta-1},
$$




$$
T_{f}=\text { (Baryon Number - Lepton Number), where } f=\{Q, \bar{u}, \bar{d}, L, \bar{e}, \bar{\nu}\} .
$$
matrix is

In the basis $\left\{\nu_{1}, \nu_{2}, \nu_{3}, \bar{\nu}_{1}, \bar{\nu}_{2}, \bar{\nu}_{3}, N_{1}, N_{2}, N_{3}\right\}$, the generalized neutrino mass

$$
M_{\nu, \bar{\nu}, N}=\left(\begin{array}{ccc}
0 & M_{D}^{\nu} & 0 \\
\left(M_{D}^{\nu}\right)^{T} & 0 & M_{\bar{\nu}, N} \\
0 & \left(M_{\bar{\nu}, N}\right)^{T} & M_{N}
\end{array}\right),
$$

where

$$
\begin{gathered}
\left(M_{D}^{\nu}\right)_{i j}=\left(Y_{\nu}\right)_{i j} \frac{v}{\sqrt{2}} \sin \beta \\
M_{\bar{\nu}, N}=\left(\begin{array}{ccc}
3 \kappa_{1} \epsilon \tilde{V}_{\bar{\psi}_{H}} & \left(\epsilon^{\prime}+3 \kappa_{2} \epsilon\right) \tilde{V}_{\bar{\psi}_{H}} & 0 \\
-\left(\epsilon^{\prime}-3 \kappa_{2} \epsilon\right) \tilde{V}_{\bar{\psi}_{H}} & 3 \epsilon \tilde{V}_{\bar{\psi}_{H}} & 0 \\
0 & r \epsilon(1-\delta) T_{\bar{\nu}} \tilde{V}_{\bar{\psi}_{H}} & \tilde{V}_{\bar{\psi}_{H}}^{\prime}
\end{array}\right), \\
M_{N}=\left(\begin{array}{ccc}
\kappa_{1} \tilde{S} & \kappa_{2} \tilde{S} & 0 \\
\kappa_{2} \tilde{S} & \tilde{S} & \tilde{\phi} \\
0 & \tilde{\phi} & 0
\end{array}\right),
\end{gathered}
$$

where $\tilde{V}_{\bar{\psi}_{H}}$ and $\tilde{V}_{\bar{\psi}_{H}}^{\prime}$ are proportional to the VEV of $\bar{\psi}_{H}$ (with different implicit Yukawa couplings), $\tilde{S}$ and $\tilde{\phi}$ are up to couplings the VEVs of $S^{22}$ and $\phi^{2}$, respectively.

It has been shown in Ref. [12] that the very good fits to the Standard Model fermion (quark, lepton, and neutrino) masses and mixings are obtained from the Yukawa matrices given in Eqs. (71-74) and the generalized neutrino mass matrix given in Eq. (77). In particular, the neutrino mixings can be bilarge, which explain the solar neutrino experiment, the atmospheric neutrino experiment, and the Kamland experiment.

\subsection{Model II}

In Model II, the zero modes $\left(\Sigma_{i}\right)_{(\mathbf{1 6},-\mathbf{3})}^{(0)}$ of $\left(\Sigma_{i}\right)_{(\mathbf{1 6},-\mathbf{3})}$ where $i=2,3$ are considered as the first two families of fermions, and the $R$ symmetry $S O(2)_{56} \times U(1)_{\boldsymbol{4}_{+}} \times S U(2)_{\boldsymbol{4}_{-}}$ can give us the flavour symmetry to generate the fermion masses and mixings.

To be convenient in discussions, we define

$$
\mathbf{1 6} \mathbf{1}_{1} \equiv-\left(\Sigma_{3}\right)_{(\mathbf{1 6},-3)}^{(0)}, \quad \mathbf{1 6} \mathbf{2}_{2} \equiv\left(\Sigma_{2}\right)_{(\mathbf{1 6},-3)}^{(0)}, \Upsilon_{0} \equiv\left(\Sigma_{1}\right)_{(\mathbf{1 6},-3)}^{(0)},
$$

where $\mathbf{1 6}_{i}$ is the $i-$ th family of the Standard Model fermions. In this convention, the first two families $\left(\mathbf{1 6}_{1}, \mathbf{1 6}_{2}\right)^{T}$ form a fundamental representation under the $S U(2)_{\mathbf{4}_{-}}$ $R$ symmetry. 
We define the $U(1)_{R}^{I I}$ and $U(1)_{F}^{I I}$ global symmetries from the $S O(2)_{56} \times U(1)_{4_{+}}$ $R$ symmetry, respectively. The generators for $U(1)_{R}^{I I}$ and $U(1)_{F}^{I I}$ in $S U(4)_{R}$ are

$$
\begin{gathered}
T_{U(1)_{R}^{I I}}=\operatorname{diag}[-1,1,0,0], \\
T_{U(1)_{F}^{I I}}=\operatorname{diag}\left[\frac{1}{2}, \frac{1}{2},-\frac{1}{2},-\frac{1}{2}\right] .
\end{gathered}
$$

So, under $U(1)_{R}^{I I}$, the Grassmann coordinate $\theta$ has charge $+1, \mathbf{1 6}_{1}$ and $\mathbf{1 6}_{2}$ have charge +1 , while $\Upsilon_{0}$ has charge 0 . In addition, under $U(1)_{F}^{I I}$, the Grassmann coordinate $\theta$ has charge $-1 / 2, \mathbf{1 6}_{1}$ and $\mathbf{1 6}_{2}$ have charge 0 , while $\Upsilon_{0}$ has charge -1 .

On the observable 3 -brane at $z=0$, we introduce the third family of fermion $16_{3}$ whose quantum numbers under $U(1)_{X} \times U(1)_{R}^{I I} \times U(1)_{F}^{I I}$ are $(-3,+1,-1 / 2)$, and a 16 spinor representation $\Upsilon_{0}^{\prime}$ whose quantum numbers under $U(1)_{X} \times U(1)_{R}^{I I} \times U(1)_{F}^{I I}$ are $(+3,+2,0)$. So, we can introduce the mass term $M_{\Upsilon_{0}} \Upsilon_{0} \Upsilon_{0}^{\prime}$ for $\Upsilon_{0}$ and $\Upsilon_{0}^{\prime}$ in the superpotential on the 3-brane at $z=0$. The mass term for $\Upsilon_{0}$ and $\Upsilon_{0}^{\prime}$ can also be generated by the superpotential $s_{1} s_{1}^{\prime} \Upsilon_{0} \Upsilon_{0}^{\prime}$ where $s_{1}$ and $s_{1}^{\prime}$ will be defined when we discuss the anomaly cancellations.

In Model II, the $R$ symmetry $U(1)_{F}^{I} \times S U(2)_{4_{-}}$can be considered as flavour symmetry to explain the fermion masses and mixings. Similar to the discussions in Model I, we assume that the particles, which are introduced to break the gauge symmetry and generate the fermion masses and mixings, are on the observable 3-brane at $z=0$. The additional particle content in Model II is the same as that in Model I. In short, the particles in Model II are the Standard Model fermions $\mathbf{1 6}_{a^{\prime}}, \mathbf{1 6}_{3}$; Higgs fields $H, 45_{H}, \psi_{H}, \bar{\psi}_{H}$; vector like particles $\Upsilon_{0}, \Upsilon_{0}^{\prime}, \psi, \bar{\psi}, \psi^{a^{\prime}}, \bar{\psi}_{a^{\prime}}, \psi_{a^{\prime}}, \bar{\psi}^{a^{\prime}}$; three $S O(10)$ singlets $N_{a^{\prime}}$ and $N_{3}$; and Higgs fields for flavour symmetry breaking $\phi^{a^{\prime}}, S^{a^{\prime} b^{\prime}}$, and $A^{a^{\prime} b^{\prime}}$. The quantum numbers for the particles in Model II under the $U(1)_{X}$ gauge symmetry and $U(1)_{R}^{I I} \times U(1)_{F}^{I I} R$ symmetry are given in Table 2 , where $Q_{X}$ and $Q_{F}$ are free parameters.

\section{Low Energy $R$ Symmetry.}

The $U(1)_{R}^{I I}$ is the traditional $U(1)_{R} R$ symmetry defined in the low energy supersymmetry phenomenology. So, the superpartners of the ordinary particles can be the candidates of cold dark matter, for instance, the neutralino.

\section{Anomaly Cancellations.}

Similar to the discussions in the subsection 3.1, the anomalies $[S O(10)]^{2} U(1)_{X}$, $\left[U(1)_{X}\right]^{3}$, and $[\text { Gravity }]^{2} U(1)_{X}$ can be cancelled by a generalized Green-Schwarz mechanism. Here, we discuss the anomaly cancellations by introducing the vector-like particles on three 3-branes at three fixed points.

Similar to the anomaly discussions in Model I, the anomalies localized on the 3-brane at each fixed point due to the bulk vector multiplet are equivalent to the 
anomalies from a multiplet with quantum number $(\mathbf{1 6}, \mathbf{- 3})$ under the $S O(10) \times U(1)_{X}$ gauge symmetry. If we consider the anomaly inflows [37, 38], like the 5-dimensional orbifold theories, the 4-dimensional anomaly cancellations are sufficient to ensure the consistency of higher dimensional orbifold theories [37]. Thus, we only need to consider the total anomalies due to the bulk zero modes and the particles localized on the 3-branes at the fixed points.

To be concrete, we still discuss the anomaly cancellations on the 3-brane at each fixed point, which are similar to those in Model I. For simplicity, we consider the case with $Q_{X}=0$.

First, on the observable 3-brane at $z=0$, the anomalies due to the zero modes of bulk vector multiplet are cancelled by the Higgs field $\bar{\psi}_{H}$. And to cancel the anomalies generated by the Higgs field $H$, we introduce one pair of 10 -plets $T_{10}$ and $T_{10}^{\prime}$ whose quantum numbers are $(\mathbf{1 0}, \mathbf{- 6})$ and $(\mathbf{1 0}, \mathbf{0})$ respectively under the $S O(10) \times U(1)_{X}$ gauge symmetry. In order to give the masses to $T_{10}$ and $T_{10}^{\prime}$, and keep the $U(1)_{X}$ D-flatness and anomaly free on the 3 -brane at $z=0$, we introduce the $S O(10)$ singlets $s_{1}$ and $s_{1}^{\prime}$ with $U(1)_{X}$ charges +6 and -6 , respectively. Moreover, to forbid the mixings between the Higgs $H$ and $T_{10}$ or between $H$ and $T_{10}^{\prime}$, we assign the following $U(1)_{R}^{I I} \times U(1)_{F}^{I I}$ charges for the vector-like particles: the $U(1)_{R}^{I I}$ charges for $T_{10}, T_{10}^{\prime}, s_{1}$ and $s_{1}^{\prime}$ are $1,1,0$ and 0 , respectively, and the $U(1)_{F}^{I I}$ charges for $T_{10}, T_{10}^{\prime}, s_{1}$ and $s_{1}^{\prime}$ are $-1 / 2,-1 / 2,0$ and 0 , respectively. In short, the anomalies $[S O(10)]^{2} U(1)_{X},\left[U(1)_{X}\right]^{3}$, and $[\text { Gravity }]^{2} U(1)_{X}$ are cancelled on the 3 -brane at $z=0$. And the masses of $T_{10}$ and $T_{10}^{\prime}$ can be generated by the superpotential $s_{1} T_{10} T_{10}^{\prime}$.

Second, on the 3 -brane at $z=\pi R e^{\mathrm{i} \pi / 6} / \sqrt{3}$, we need to cancel the anomalies generated by a multiplet with quantum number $(\mathbf{1 6}, \mathbf{- 3})$ under the $S O(10) \times U(1)_{X}$ gauge symmetry. So, we introduce a pair of the $\Upsilon_{2}$ and $\Upsilon_{2}^{\prime}$ whose quantum numbers are $(\mathbf{1 6}, \mathbf{0})$ and $(\overline{\mathbf{1 6}},+\mathbf{3})$ respectively under the $S O(10) \times U(1)_{X}$ gauge symmetry. And in order to give the masses to $\Upsilon_{2}$ and $\Upsilon_{2}^{\prime}$, and keep the $U(1)_{X}$ D-flatness and anomaly free on the 3 -brane at $z=\pi R e^{\mathrm{i} \pi / 6} / \sqrt{3}$, we introduce the $S O(10)$ singlets $s_{2}$ and $s_{2}^{\prime}$ with $U(1)_{X}$ charges -3 and +3 , respectively. In addition, to forbid the mixings between the SM fermions $\mathbf{1 6}_{j}(j=1,2)$ and $\Upsilon_{2}$, and the mass terms between $\mathbf{1 6}_{j}$ and $\Upsilon_{2}^{\prime}$, we assign the following $U(1)_{R}^{I I} \times U(1)_{F}^{I I}$ charges for the vector-like particles: the $U(1)_{R}^{I I}$ charges for $\Upsilon_{2}, \Upsilon_{2}^{\prime}, s_{2}$ and $s_{2}^{\prime}$ are 1, 1, 0 and 0 , respectively, and the $U(1)_{F}^{I I}$ charges for $\Upsilon_{2}, \Upsilon_{2}^{\prime}, s_{2}$ and $s_{2}^{\prime}$ are $-1 / 2,-1 / 2,0$ and 0 , respectively. And the masses of $\Upsilon_{2}$ and $\Upsilon_{2}^{\prime}$ can be generated by the superpotential $s_{2} \Upsilon_{2} \Upsilon_{2}^{\prime}$.

Third, the anomaly cancellations on the 3-brane at $z=2 \pi R e^{\mathrm{i} \pi / 6} / \sqrt{3}$ is similar to those on the 3-brane at $z=\pi R e^{\mathrm{i} \pi / 6} / \sqrt{3}$. We introduce the particles $\Upsilon_{3}, \Upsilon_{3}^{\prime}, s_{3}$ and $s_{3}^{\prime}$, whose quantum numbers are the same as those for $\Upsilon_{2}, \Upsilon_{2}^{\prime}, s_{2}$ and $s_{2}^{\prime}$, respectively.

\section{Fermion Masses and Mixings.}

With the particle quantum numbers given in Table 2, we obtain that the superpotential in Model II is the same as that in Model I given by Eq. (68). Therefore, the discussions for the fermion masses and mixings are the same as those in Model I, too. The effective Yukawa matrices for up-type quarks, down-type quarks, and 
charged leptons, and the Dirac neutrino Yukawa matrix are given by Eqs. (71-74), and the generalized neutrino mass matrix is given by Eq. (77).

In addition, we consider the cut-off scale $\left(M_{*}\right)$ is close to the compactification scale $(1 / R)$ due to the unitarity constraints [39]. Then, comparing to the Yukawa couplings of the third family, the volume suppression factor $1 /\left(M_{*} R\right)$ for the Yukawa couplings of the first two families of fermions is close to 1, i.e, at the order of $1(\mathcal{O}(1))$.

In short, we can also have very good fits to the Standard Model fermion masses and mixings, especially the neutrino mixings can be bilarge.

\section{Comments on the $\mathcal{N}=(1,1)$ Supersymmetric $E_{6}$ Models on $M^{4} \times T^{2} / Z_{4}$ and $M^{4} \times T^{2} / Z_{6}$}

We are only interested in the models where there are at least two families of the SM fermions from the zero modes of bulk vector multiplet. In this Section, we comment on the $\mathcal{N}=(1,1)$ supersymmetric $E_{6}$ models on $M^{4} \times T^{2} / Z_{n}$ in which $n=4$ and 6 .

The discussions for $E_{6}$ symmetry breaking are similar to those in the Section 3. The $R_{\omega}^{E_{6}}$ for $E_{6}$ gauge group is the product of the $R_{\omega}^{S O(10)}$ for $S O(10)$ and $R_{\omega}^{U(1)_{X}}$ for $U(1)_{X}$, and we choose

$$
\begin{gathered}
R_{\omega}^{S O(10)=} \operatorname{diag}[+1,+1,+1,+1,+1,+1,+1,+1,+1,+1], \\
R_{\omega}^{U(1)_{X}}=\exp \left(-\mathrm{i} \frac{2 \pi Q}{3 n}\right),
\end{gathered}
$$

where $n=4$ and 6 for the $T^{2} / Z_{4}$ and $T^{2} / Z_{6}$ orbifolds, respectively.

In order to have two families of the SM fermions from the zero modes of bulk vector multiplet, we obtain $k=0$. Using Eqs. (43-46) and (84-85), we obtain the transformations of vector multiplet

$$
\begin{gathered}
V_{(\mathbf{4 5}, \mathbf{0})}\left(x^{\mu}, \omega z, \omega^{-1} \bar{z}\right)=V_{(\mathbf{4 5}, \mathbf{0})}\left(x^{\mu}, z, \bar{z}\right) \\
V_{(\mathbf{1}, \mathbf{0})}\left(x^{\mu}, \omega z, \omega^{-1} \bar{z}\right)=V_{(\mathbf{1}, \mathbf{0})}\left(x^{\mu}, z, \bar{z}\right) \\
V_{(\mathbf{1 6},-\mathbf{3})}\left(x^{\mu}, \omega z, \omega^{-1} \bar{z}\right)=\omega V_{(\mathbf{1 6},-\mathbf{3})}\left(x^{\mu}, z, \bar{z}\right) \\
V_{(\overline{\mathbf{1 6}, 3)}}\left(x^{\mu}, \omega z, \omega^{-1} \bar{z}\right)=\omega^{n-1} V_{(\overline{\mathbf{1 6}}, \mathbf{3})}\left(x^{\mu}, z, \bar{z}\right), \\
\left(\Sigma_{j}\right)_{(\mathbf{4 5}, \mathbf{0})}\left(x^{\mu}, \omega z, \omega^{-1} \bar{z}\right)=\omega^{n-1}\left(\Sigma_{j}\right)_{(\mathbf{4 5}, \mathbf{0})}\left(x^{\mu}, z, \bar{z}\right), \\
\left(\Sigma_{j}\right)_{(\mathbf{1}, \mathbf{0})}\left(x^{\mu}, \omega z, \omega^{-1} \bar{z}\right)=\omega^{n-1}\left(\sum_{j}\right)_{(\mathbf{1}, \mathbf{0})}\left(x^{\mu}, z, \bar{z}\right)
\end{gathered}
$$




$$
\begin{aligned}
\left(\Sigma_{j}\right)_{(\mathbf{1 6},-\mathbf{3})}\left(x^{\mu}, \omega z, \omega^{-1} \bar{z}\right) & =\left(\Sigma_{j}\right)_{(\mathbf{1 6},-\mathbf{3})}\left(x^{\mu}, z, \bar{z}\right) \\
\left(\Sigma_{j}\right)_{\overline{\mathbf{1 6}, 3}, \mathbf{3})}\left(x^{\mu}, \omega z, \omega^{-1} \bar{z}\right) & =\omega^{n-2}\left(\Sigma_{j}\right)_{\overline{\mathbf{1 6}, 3}, \mathbf{3})}\left(x^{\mu}, z, \bar{z}\right) \\
\left(\Sigma_{3}\right)_{(\mathbf{4 5}, \mathbf{0})}\left(x^{\mu}, \omega z, \omega^{-1} \bar{z}\right) & =\omega^{2}\left(\Sigma_{3}\right)_{(\mathbf{4 5}, \mathbf{0})}\left(x^{\mu}, z, \bar{z}\right) \\
\left(\Sigma_{3}\right)_{(\mathbf{1}, \mathbf{0})}\left(x^{\mu}, \omega z, \omega^{-1} \bar{z}\right) & =\omega^{2}\left(\Sigma_{3}\right)_{(\mathbf{1}, \mathbf{0})}\left(x^{\mu}, z, \bar{z}\right) \\
\left(\Sigma_{3}\right)_{(\mathbf{1 6},-\mathbf{3})}\left(x^{\mu}, \omega z, \omega^{-1} \bar{z}\right) & =\omega^{3}\left(\Sigma_{3}\right)_{(\mathbf{1 6},-\mathbf{3})}\left(x^{\mu}, z, \bar{z}\right) \\
\left(\Sigma_{3}\right)_{(\overline{\mathbf{1 6}}, \mathbf{3})}\left(x^{\mu}, \omega z, \omega^{-1} \bar{z}\right) & =\omega\left(\Sigma_{3}\right)_{(\overline{\mathbf{1 6}}, \mathbf{3})}\left(x^{\mu}, z, \bar{z}\right)
\end{aligned}
$$

where $j=1,2$, and $\omega=e^{\mathrm{i} 2 \pi / n}(n=4,6)$.

From the general discussions on gauge symmetry breaking by discrete symmetry [26], we obtain that only the fields $V_{(\mathbf{4 5 , 0})}, V_{(\mathbf{1 , 0})},\left(\Sigma_{1}\right)_{(\mathbf{1 6},-\mathbf{3})}$ and $\left(\Sigma_{2}\right)_{(\mathbf{1 6},-\mathbf{3})}$ have zero modes. So, the $E_{6}$ gauge symmetry is broken down to $S O(10) \times U(1)_{X}$ for the zero modes by orbifold projection. And there are two $S O(10)$ spinor representations 16 from the zero modes of bulk vector multiplet, which can be considered as two families of fermions. The $R$ symmetry is $S O(2)_{56} \times U(1)_{4_{+}} \times U(1)_{4_{-}}$, which can be the origin of flavour symmetry.

We define the zero modes $\left(\Sigma_{1}\right)_{(\mathbf{1 6},-\mathbf{3})}^{(0)}$ and $\left(\Sigma_{2}\right)_{(\mathbf{1 6},-\mathbf{3})}^{(0)}$ of $\left(\Sigma_{1}\right)_{(\mathbf{1 6},-\mathbf{3})}$ and $\left(\Sigma_{2}\right)_{(\mathbf{1 6},-\mathbf{3})}$ as the first two families of fermions

$$
\mathbf{1 6}_{1} \equiv\left(\Sigma_{1}\right)_{(\mathbf{1 6},-3)}^{(0)}, \mathbf{1 6}_{2} \equiv\left(\Sigma_{2}\right)_{(\mathbf{1 6},-3)}^{(0)} .
$$

To be convenient, we define three $U(1)_{R}^{I I I}, U(1)_{F}^{I I I}$ and $U(1)_{R F}$ global symmetries from the $R$ symmetry $S O(2)_{56} \times U(1)_{4_{+}} \times U(1)_{4_{-}}$, whose generators in $S U(4)_{R}$ are

$$
\begin{aligned}
T_{U(1)_{R}^{I I I}} & =\operatorname{diag}[-1,0,0,+1], \\
T_{U(1)_{F}^{I I I}} & =\operatorname{diag}\left[0, \frac{1}{2},-\frac{1}{2}, 0\right], \\
T_{U(1)_{R F}} & =\operatorname{diag}\left[\frac{1}{2},-\frac{1}{2},-\frac{1}{2}, \frac{1}{2}\right] .
\end{aligned}
$$

Under $U(1)_{R}^{I I I}$ symmetry, the Grassmann coordinate $\theta, \mathbf{1 6}_{1}$ and $\mathbf{1 6}_{2}$ have charge +1 , so, the $U(1)_{R}^{I I I}$ is the traditional $U(1)_{R} R$ symmetry in the low energy supersymmetry phenomenology. And under $U(1)_{F}^{I I I}$ global symmetry, the Grassmann coordinate $\theta$ 
has charge $0, \mathbf{1 6}_{1}$ and $\mathbf{1 6}_{2}$ have charges $-1 / 2$ and $+1 / 2$, respectively. In addition, under $U(1)_{R F}$ global symmetry, the Grassmann coordinate $\theta$ has charge $-1 / 2$, and the $\mathbf{1 6}_{1}$ and $\mathbf{1 6}_{2}$ have charge 0 . The $U(1)_{F}^{I I I} \times U(1)_{R F} R$ symmetry can be considered as flavour symmetry.

Similar to the discussions in Section 3, the anomalies $[S O(10)]^{2} U(1)_{X},\left[U(1)_{X}\right]^{3}$, and [Gravity $]^{2} U(1)_{X}$, can be cancelled by a generalized Green-Schwarz mechanism, or by adding the suitable vector-like particles on the 3-branes at the fixed points. If we introduce the suitable vector-like particles on the 3-branes at the fixed points to cancel the anomalies, it is easier if we consider the anomaly inflows among the 3 -branes at the fixed points.

The supersymmetric $S O(10)$ models with $U(1)$ and extra discrete flavour symmetry $[9,10]$ or $U(1)_{A}$ symmetry [19] have been studied previously, and those discussions on fermion masses and mixings may be generalized to these models. However, how to explain the fermion masses and mixings in the supersymmetric $S O(10) \times U(1)_{X}$ models with $U(1)_{F}^{I I I} \times U(1)_{R F}$ flavour symmetry is still an interesting question and deserves further detail study.

\section{Comments on the $\mathcal{N}=(1,1)$ Supersymmetric $S U(9)$ and $S U(8)$ Models on $T^{2}$ Orbifolds}

In this Section, we briefly discuss the $S U(9)$ models on the space-time $M^{4} \times T^{2} / Z_{3}$ where there are three or two families of fermions from the zero modes of bulk vector multiplet. We also briefly discuss the $S U(8)$ Models on the space-times $M^{4} \times T^{2} / Z_{3}$, $M^{4} \times T^{2} / Z_{4}$ and $M^{4} \times T^{2} / Z_{6}$ where there are three or two families from the zero modes of bulk vector multiplet.

\subsection{The $S U(9)$ Models on $M^{4} \times T^{2} / Z_{3}$}

These models have been briefly commented in Ref. [33]. In order to break the $S U(9)$ gauge symmetry, we choose $R_{\omega}$ in the adjoint representation of $S U(9)$

$$
R_{\omega}^{S U(9)}=\operatorname{diag}\left[+1,+1,+1, \omega^{2}, \omega^{2}, \omega^{2}, \omega, \omega, \omega\right]
$$

where $\omega=e^{\mathrm{i} 2 \pi / 3}$.

Under $R_{\omega}^{S U(9)}$, the $S U(9)$ gauge symmetry is broken down to the $S U(3)_{C} \times$

$S U(3)_{L} \times S U(3)_{R} \times U(1) \times U(1)^{\prime}$ gauge symmetry. And the generators for $U(1)$ and $U(1)^{\prime}$ are

$$
\begin{gathered}
T_{U(1)}=\operatorname{diag}[+1,+1,+1,-1,-1,-1,0,0,0], \\
T_{U(1)^{\prime}}=\operatorname{diag}[+1,+1,+1,+1,+1,+1,-2,-2,-2] .
\end{gathered}
$$


Under the $S U(3)_{C} \times S U(3)_{L} \times S U(3)_{R} \times U(1) \times U(1)^{\prime}$ gauge symmetry, the $S U(9)$ adjoint representation $\mathbf{8 0}$ is decomposed as

$$
80=\mathrm{MG}(78)+(\mathbf{1}, 1,1)_{(0,0)}+(\mathbf{1}, \mathbf{1}, \mathbf{1})_{(0,0)},
$$

where

$$
\operatorname{MG}(\mathbf{7 8})=\left(\begin{array}{ccc}
(\mathbf{8}, \mathbf{1}, \mathbf{1})_{(\mathbf{0}, 0)} & (3, \overline{3}, \mathbf{1})_{(2,0)} & (\mathbf{3}, 1, \overline{\mathbf{3}})_{(1,3)} \\
(\overline{\mathbf{3}}, \mathbf{3}, \mathbf{1})_{(-2,0)} & (\mathbf{1}, \mathbf{8}, \mathbf{1})_{(\mathbf{0}, 0)} & (\mathbf{1}, \mathbf{3}, \overline{\mathbf{3}})_{(-1,3)} \\
(\overline{\mathbf{3}}, \mathbf{1}, \mathbf{3})_{(-1,-3)} & (\mathbf{1}, \overline{\mathbf{3}}, \mathbf{3})_{(1,-3)} & (\mathbf{1}, \mathbf{1}, \mathbf{8})_{(\mathbf{0}, 0)}
\end{array}\right),
$$

where the subscripts denote the charges under the $U(1) \times U(1)^{\prime}$ gauge symmetry.

Note that $k=0$ for $T^{2} / Z_{3}$ orbifold. Using Eqs. (43-46) and (102), we obtain that the $Z_{3}$ transformation properties for these decomposed representations of the vector multiplet $V$ and chiral multiplets $\Sigma_{i}$ are

$$
V:\left(\begin{array}{ccc}
1 & \omega & \omega^{2} \\
\omega^{2} & 1 & \omega \\
\omega & \omega^{2} & 1
\end{array}\right)+(1)+(1), \quad \Sigma_{i}:\left(\begin{array}{ccc}
\omega^{2} & 1 & \omega \\
\omega & \omega^{2} & 1 \\
1 & \omega & \omega^{2}
\end{array}\right)+\left(\omega^{2}\right)+\left(\omega^{2}\right) .
$$

So, the $(\mathbf{3}, \overline{\mathbf{3}}, \mathbf{1})_{(\mathbf{2}, \mathbf{0})},(\mathbf{1}, \mathbf{3}, \overline{\mathbf{3}})_{(-\mathbf{1}, \mathbf{3})}$, and $(\overline{\mathbf{3}}, \mathbf{1}, \mathbf{3})_{(-\mathbf{1},-\mathbf{3})}$ in $\Sigma_{i}(i=1,2,3)$ have the zero modes. And these zero modes can be considered as three families or two families of fermions in the "trinification".

Similar to the discussions in Section 3, we can obtain the $S U(9)$ model with $U(1)_{F}^{I} \times S U(2)_{4}$ flavour symmetry and three families of fermions from the zero modes of bulk vector multiplet, and the $S U(9)$ model with $U(1)_{F}^{I I} \times S U(2)_{4_{-}}$flavour symmetry and two families from the zero modes of bulk vector multiplet. Therefore, how to explain the fermion masses and mixings in the extra "trinification" $S U(3)_{C} \times$ $S U(3)_{L} \times S U(3)_{R} \times U(1) \times U(1)^{\prime}$ with these flavour symmetries is another interesting question. By the way, the anomalies due to the $U(1) \times U(1)^{\prime}$ gauge symmetry can be cancelled similarly.

\subsection{The $S U(8)$ Models on $M^{4} \times T^{2} / Z_{n}$}

The $S U(8)$ model on $M^{4} \times T^{2} / Z_{6}$ has been discussed previously where the electroweak (EW) breaking Higgs fields as well as the third family of fermions are unified into the 6-dimensional vector multiplet [28]. Here, we concentrate on the models with three or two families of fermions from the zero modes of bulk vector multiplet.

I. $S U(8)$ Models on $M^{4} \times T^{2} / Z_{3}$.

In order to break the $S U(8)$ gauge symmetry, we choose $R_{\omega}$ in the adjoint representation of $S U(8)$

$$
R_{\omega}^{S U(8)}=\operatorname{diag}\left[+1,+1,+1,+1, \omega^{2}, \omega^{2}, \omega, \omega\right],
$$


where $\omega=e^{\mathrm{i} 2 \pi / 3}$.

Under $R_{\omega}^{S U(8)}$, the $S U(8)$ gauge symmetry is broken down to the $S U(4)_{C} \times$ $S U(2)_{L} \times S U(2)_{R} \times U(1) \times U(1)^{\prime}$ gauge symmetry. And the generators for $U(1)$ and $U(1)^{\prime}$ are

$$
\begin{gathered}
T_{U(1)}=\operatorname{diag}[+1,+1,+1,+1,-1,-1,-1,-1], \\
T_{U(1)^{\prime}}=\operatorname{diag}[0,0,0,0,+1,+1,-1,-1] .
\end{gathered}
$$

Under the $S U(4)_{C} \times S U(2)_{L} \times S U(2)_{R} \times U(1) \times U(1)^{\prime}$ gauge symmetry, the $S U(8)$ adjoint representation $\mathbf{6 3}$ is decomposed as

$$
63=\operatorname{MG}(61)+(1,1,1)_{(0,0)}+(1,1,1)_{(0,0)},
$$

where

$$
\operatorname{MG}(61)=\left(\begin{array}{ccc}
(15,1,1)_{(0,0)} & (4,2,1)_{(2,-1)} & (4,1,2)_{(2,1)} \\
(\overline{4}, 2,1)_{(-2,1)} & (1,3,1)_{(0,0)} & (1,2,2)_{(0,2)} \\
(\overline{4}, 1,2)_{(-2,-1)} & (1,2,2)_{(0,-2)} & (1,1,3)_{(0,0)}
\end{array}\right)
$$

where the subscripts denote the charges under the $U(1) \times U(1)^{\prime}$ gauge symmetry.

Note that $k=0$ for $T^{2} / Z_{3}$ orbifold. Using Eqs. (43-46) and (108), we obtain that the $Z_{3}$ transformation properties for these decomposed representations of the vector multiplet $V$ and chiral multiplets $\Sigma_{i}$ are

$$
V:\left(\begin{array}{ccc}
1 & \omega & \omega^{2} \\
\omega^{2} & 1 & \omega \\
\omega & \omega^{2} & 1
\end{array}\right)+(1)+(1), \quad \Sigma_{i}:\left(\begin{array}{ccc}
\omega^{2} & 1 & \omega \\
\omega & \omega^{2} & 1 \\
1 & \omega & \omega^{2}
\end{array}\right)+\left(\omega^{2}\right)+\left(\omega^{2}\right) .
$$

So, the $(\mathbf{4}, \mathbf{2}, \mathbf{1})_{(\mathbf{2},-\mathbf{1})},(\overline{\mathbf{4}}, \mathbf{1}, \mathbf{2})_{(-\mathbf{2},-\mathbf{1})}$ and $(\mathbf{1}, \mathbf{2}, \mathbf{2})_{(\mathbf{0}, \mathbf{2})}$ in $\Sigma_{i}(i=1,2,3)$ have the zero modes. The zero modes of $(\mathbf{4}, \mathbf{2}, \mathbf{1})_{(\mathbf{2},-\mathbf{1})}$ and $(\overline{\mathbf{4}}, \mathbf{1}, \mathbf{2})_{(-\mathbf{2},-\mathbf{1})}$ in $\Sigma_{i}$ can be considered as three families of the SM fermions in the Pati-Salam model, while the $(\mathbf{1}, \mathbf{2}, \mathbf{2})_{(\mathbf{0}, \mathbf{2})}$ in $\Sigma_{i}$ can be considered as three bi-doublet EW breaking Higgs particles. Unfortunately, these Higgs fields can not give the correct masses and mixings for the Standard Model fermions unless we add various Yukawa terms on the 3-branes at the fixed points, so, we do not consider them as the EW breaking Higgs particles, i.e., we give up the gauge-Higgs-fermion unification or the gauge-Yukawa unification. For simplicity, we introduce the Standard Model EW breaking Higgs fields on the observable 3-brane at the fixed point $z=0$, which are similar to those in Section 3.

Similar to the discussions in Section 3, we can have the $S U(8)$ model with $U(1)_{F}^{I} \times S U(2)_{4_{-}}$flavour symmetry and three families of fermions from the zero modes of bulk vector multiplet, and the $S U(8)$ model with $U(1)_{F}^{I I} \times S U(2)_{4}$ flavour symmetry and two families of fermions from the zero modes of bulk vector multiplet. Essentially speaking, the $S O(10)$ models are similar to the Pati-Salam models when 
we consider the fermion masses and mixings. Thus, similar to the discussions in Section 3, we can explain the fermion masses and mixings via these flavour symmetries. And three bi-doublets from the zero modes of bulk vector multiplet can obtain the heavy masses if we couple them with the singlets which have non-zero VEVs on the 3-branes at the fixed points. By the way, the discussions for anomaly cancellations due to the $U(1) \times U(1)^{\prime}$ gauge symmetry are similar to those in Section 3 , too.

\section{II. $S U(8)$ Models on $M^{4} \times T^{2} / Z_{4}$ and $M^{4} \times T^{2} / Z_{6}$.}

We consider the $S U(8)$ Models on the space-time $M^{4} \times T^{2} / Z_{n}$ where $n=4,6$. In order to break the $S U(8)$ gauge symmetry, we choose

$$
R_{\omega}^{S U(8)}=\operatorname{diag}\left[+1,+1,+1,+1, \omega^{n-1}, \omega^{n-1}, \omega, \omega\right]
$$

where $\omega=e^{\mathrm{i} 2 \pi / n}$ with $n=4,6$. Under $R_{\omega}^{S U(8)}$, the $S U(8)$ gauge symmetry is broken down to the $S U(4)_{C} \times S U(2)_{L} \times S U(2)_{R} \times U(1) \times U(1)^{\prime}$ gauge symmetry.

Using Eqs. (43-46) with $k=0$ and Eq. (114), we obtain that the $Z_{n}$ transformation properties for these decomposed representations of the vector multiplet $V$ and chiral multiplets $\Sigma_{i}$ are

$$
\begin{gathered}
V:\left(\begin{array}{ccc}
1 & \omega & \omega^{n-1} \\
\omega^{n-1} & 1 & \omega^{n-2} \\
\omega & \omega^{2} & 1
\end{array}\right)+(1)+(1), \quad \Sigma_{3}:\left(\begin{array}{ccc}
\omega^{2} & \omega^{3} & \omega \\
\omega & \omega^{2} & 1 \\
\omega^{3} & \omega^{4} & \omega^{2}
\end{array}\right)+\left(\omega^{2}\right)+\left(\omega^{2}\right), \\
\Sigma_{j}:\left(\begin{array}{ccc}
\omega^{n-1} & 1 & \omega^{n-2} \\
\omega^{n-2} & \omega^{n-1} & \omega^{n-3} \\
1 & \omega & \omega^{n-1}
\end{array}\right)+\left(\omega^{n-1}\right)+\left(\omega^{n-1}\right),
\end{gathered}
$$

where $j=1,2$. So, the $(\mathbf{4}, \mathbf{2}, \mathbf{1})_{(\mathbf{2},-\mathbf{1})}$ and $(\overline{\mathbf{4}}, \mathbf{1}, \mathbf{2})_{(-\mathbf{2},-\mathbf{1})}$ in $\Sigma_{j}(j=1,2)$ have zero modes, which can be considered as two families of fermions in the Pati-Salam model. The $(\mathbf{1}, \mathbf{2}, \mathbf{2})_{(\mathbf{0 , 2})}$ and $(\mathbf{1}, \mathbf{2}, \mathbf{2})_{(\mathbf{0},-\mathbf{2})}$ in $\Sigma_{3}$ for $T^{2} / Z_{4}$ orbifold, and the $(\mathbf{1}, \mathbf{2}, \mathbf{2})_{(\mathbf{0 , 2})}$ in $\Sigma_{3}$ for $T^{2} / Z_{6}$ orbifold have zero modes, too. The $(\mathbf{1}, \mathbf{2}, \mathbf{2})_{(\mathbf{0 , 2})}$ in $\Sigma_{3}$ can be considered as a bi-doublet Higgs particle. Similar to the $S U(8)$ models on $M^{4} \times T^{2} / Z_{3}$, this bi-doublet Higgs particle can not give the correct Standard Model fermion masses and mixings unless we add various Yukawa terms on the 3-branes at the fixed points, so, we do not consider it as the EW breaking Higgs particle.

Similar to the discussions in Section 4, we can have $U(1)_{F}^{I I I} \times U(1)_{R F} R$ symmetry which can be considered as flavour symmetry. Because the $S O(10)$ models are similar to the Pati-Salam models when we consider the fermion masses and mixings, like the models in Section 4, how to explain the fermion masses and mixings by this flavour symmetry deserves further study in detail.

\section{Discussions and Conclusions}

The other interesting flavour symmetry is $S U(3)$. There are two possibilities for $S U(3)$ flavour symmetry: one is the gauged flavour symmetry, the other is the global 
flavour symmetry. For the gauged $S U(3)$ flavour symmetry, one can consider the GUT group with rank 7 or higher, for example $E_{8}$ [29]. And for the global $S U(3)$ flavour symmetry, we should consider the 10-dimensional space-time if this $S U(3)$ flavour symmetry arises from the $R$ symmetry. In the 10 -dimensional $\mathcal{N}=1$ supersymmetry, the $S O(6)(S O(9,1) \longrightarrow S O(3,1) \times S O(6))$ rotation on the extra 6-dimensional space manifold becomes the $R$ symmetry after compactification. Note that the Lie algebra of $S O(6)$ is isomorphic to that of $S U(4)$ and we only keep the 4-dimensional $\mathcal{N}=1$ supersymmetry after compactification, similar to the discussions in Sections 2 and 3 , the $S U(4)_{R} / U(1)_{R}$ or $S U(3)_{R} R$ symmetry may become the flavour symmetry if we consider the $T^{6} / Z_{3}$ orbifold. However, the 10 -dimensional $\mathcal{N}=1$ supersymmetric non-abelian gauge theory generically has gauge anomaly, and can be cancelled if and only if the gauge groups are $S O(32), E_{8} \times E_{8}, E_{8} \times U(1)^{248}$ and $U(1)^{496}$. Thus, it is difficult to construct the GUT models with global $S U(3)$ flavour symmetry from $R$ symmetry because it is not easy to break these GUT groups down to the Standard Model gauge group.

In this paper, we discuss the 6 -dimensional $\mathcal{N}=(1,1)$ supersymmetric gauge theory on the space-time $M^{4} \times T^{2}$, where the $R$ symmetry is $S O(2)_{56} \times S U(2)_{4_{+}} \times$ $S U(2)_{4_{-}}$. In order to preserve only the 4 -dimensional $\mathcal{N}=1$ supersymmetry, we consider the supersymmetry breaking and $R$ symmetry breaking on the $T^{2} / Z_{n}$ orbifolds. We find that for $T^{2} / Z_{3}$ orbifold, the only possibility is $k=0$ and the $R$ symmetry is $S O(2)_{56} \times U(1)_{4_{+}} \times S U(2)_{4_{-}}$. For $T^{2} / Z_{4}$ orbifold, the only possibility is $k=0$, too. And for $T^{2} / Z_{6}$ orbifold, there are two possibilities: $k=0$ and $k=1$. The $R$ symmetry for the $T^{2} / Z_{4}$ and $T^{2} / Z_{6}$ orbifolds is $S O(2)_{56} \times U(1)_{4_{+}} \times U(1)_{4_{-}}$. We also present the transformation properties of the bulk vector multiplet under these $R$ symmetries.

In the 6 -dimensional $\mathcal{N}=(1,1)$ supersymmetric gauge theory, the Standard Model fermions may come from the zero modes of bulk vector multiplet due to the orbifold gauge symmetry breaking, which may explain why there are three families of fermions in the Standard Model. And the $R$ symmetry can give us the flavour symmetry to explain the fermion masses and mixings. To be concrete, we discuss the $\mathcal{N}=(1,1)$ supersymmetric $E_{6}$ models on the space-time $M^{4} \times T^{2} / Z_{3}$ where the gauge symmetry $E_{6}$ is broken down to $S O(10) \times U(1)_{X}$ by orbifold projection. There are three $S O(10)$ spinor representation $\mathbf{1 6}$ s from the zero modes of bulk vector multiplet, which can be considered as three families of fermions. We study two models in detail. In Model I, three families of the Standard Model fermions arise from the zero modes of bulk vector multiplet, and the $R$ symmetry $U(1)_{F}^{I} \times S U(2)_{4_{-}}$can be considered as flavour symmetry. However, the $U(1)_{R}^{I} R$ symmetry is not the traditional $U(1)_{R}$ $R$ symmetry in the low energy supersymmetry phenomenology, and then, there are no cold dark matter candidates (for example neutralino) from the superpartners. In Model II, the first two families of the Standard Model fermions arise from the zero modes of bulk vector multiplet, and the $R$ symmetry $U(1)_{F}^{I I} \times S U(2)_{4_{-}}$can be considered as flavour symmetry. The $U(1)_{R}^{I I} R$ symmetry is the traditional $U(1)_{R}$ $R$ symmetry in the low energy supersymmetry phenomenology. In these models, the anomalies can be cancelled, and we have very good fits to the Standard Model fermion 
masses and mixings.

Furthermore, we comment on the $\mathcal{N}=(1,1)$ supersymmetric $E_{6}$ models on $M^{4} \times T^{2} / Z_{4}$ and $M^{4} \times T^{2} / Z_{6}$ where there are two families of the Standard Model fermions from the zero modes of bulk vector multiplet. We also comment on the $\mathcal{N}=(1,1)$ supersymmetric $S U(9)$ models on $M^{4} \times T^{2} / Z_{3}$, and the $S U(8)$ models on $M^{4} \times T^{2} / Z_{n}$ orbifolds in which $n=3,4,6$.

\section{Acknowledgments}

We would like to thank S. Adler and S. Nandi for helpful discussions. This research was supported by the National Science Foundation under Grant No. PHY-0070928. 


\section{References}

[1] S. Dimopoulos and F. Wilczek, NSF-ITP-82-07 (1981).

[2] Q. R. Ahmad et al. [SNO Collaboration], Phys. Rev. Lett. 89 (2002) 011301; Phys. Rev. Lett. 89 (2002) 011302.

[3] Y. Fukuda et al. [Super-Kamiokande Collaboration], Phys. Rev. Lett. 81 (1998) 1562 .

[4] K. Eguchi et al. [KamLAND Collaboration], Phys. Rev. Lett. 90 (2003) 021802.

[5] P. C. de Holanda and A.Yu. Smirnov, JCAP 0302, 001 (2003); A.Yu. Smirnov, hep-ph/0305106.

[6] V. Barger and D. Marfatia, Phys. Lett. B 555, 144 (2003).

[7] M. Apollonio et al.[CHOOZ Collaboration], Phys. Lett. B466 (1999) 415.

[8] C. D. Froggatt and H. B. Nielsen, Nucl. Phys. B147 (1979) 277.

[9] C. H. Albright and S. M. Barr, Phys. Rev. D 58, 013002 (1998); Phys. Lett. B 452, 287 (1999); Phys. Rev. Lett. 85, 244 (2000); Phys. Rev. D 62, 093008 (2000); Phys. Rev. D 64, 073010 (2001); Phys. Rev. D 67, 013002 (2003); C. H. Albright, K. S. Babu and S. M. Barr, Phys. Rev. Lett. 81, 1167 (1998).

[10] K. S. Babu, J. C. Pati and F. Wilczek, Nucl. Phys. B 566, 33 (2000).

[11] R. Barbieri, L. J. Hall, S. Raby and A. Romanino, Nucl. Phys. B 493, 3 (1997); R. Barbieri, L. J. Hall and A. Romanino, Phys. Lett. B 401, 47 (1997); R. Barbieri, L. Giusti, L. J. Hall and A. Romanino, Nucl. Phys. B 550, 32 (1999).

[12] T. Blazek, S. Raby and K. Tobe, Phys. Rev. D 60, 113001 (1999); Phys. Rev. D 62, 055001 (2000).

[13] S. Raby, Phys. Lett. B 561, 119 (2003).

[14] M. C. Chen and K. T. Mahanthappa, Phys. Rev. D 62, 113007 (2000); Phys. Rev. D 65, 053010 (2002); Phys. Rev. D 68, 017301 (2003).

[15] R. Kitano and Y. Mimura, Phys. Rev. D 63, 016008 (2001).

[16] Z. Berezhiani and A. Rossi, JHEP 9903, 002 (1999); G. G. Ross and L. VelascoSevilla, Nucl. Phys. B 653, 3 (2003); S. F. King and G. G. Ross, Phys. Lett. B 520, 243 (2001); hep-ph/0307190.

[17] R. Kitano and T. Li, Phys. Rev. D 67, 116004 (2003).

[18] Q. Shafi and Z. Tavartkiladze, Phys. Lett. B 487, 145 (2000).

[19] N. Maekawa, Prog. Theor. Phys. 106, 401 (2001).

[20] Q. Shafi and Z. Tavartkiladze, Nucl. Phys. B 665, 469 (2003). 
[21] N. Haba and Y. Shimizu, hep-ph/0210146; N. Haba, T. Kondo and Y. Shimizu, Phys. Lett. B 531, 245 (2002); Phys. Lett. B 535, 271 (2002).

[22] M. C. Chen and K. T. Mahanthappa, hep-ph/0305088.

[23] Y. Kawamura, Prog. Theor. Phys. 103 (2000) 613 hep-ph/9902423, hepph/0012125, hep-ph/0012352; G. Altarelli and F. Feruglio, hep-ph/0102301; L. Hall and Y. Nomura, hep-ph/0103125; T. Kawamoto and Y. Kawamura, hep-ph/0106163; A. Hebecker and J. March-Russell, hep-ph/0106166, hepph/0107039; R. Barbieri, L. Hall and Y. Nomura, hep-ph/0106190, hepth/0107004; A. B. Kobakhidze, hep-ph/0102323; T. Li, hep-th/0107136, hepph/0108120; hep-ph/0108238; J. A. Bagger, F. Feruglio and F. Zwirner, hepth/0107128; A. Masiero, C. A. Scrucca, M. Serone and L. Silvestrini, hepph/0107201; L. Hall, H. Murayama and Y. Nomura, hep-th/0107245; C. Csaki, G. D. Kribs and J. Terning, hep-ph/0107266; L. Hall, Y. Nomura and D. Smith, hep-ph/0107331; N. Maru, hep-ph/0108002; T. Asaka, W. Buchmuller and L. Covi, hep-ph/0108021; L. Hall, Y. Nomura, T. Okui and D. Smith, hepph/0108071; N. Haba, T. Kondo, Y. Shimizu, T. Suzuki and K. Ukai, hepph/0108003; L. Hall, J. March-Russell, T. Okui and D. Smith, hep-ph/0108161; R. Dermisek and A. Mafi, hep-ph/0108139; T. Watari and T. Yanagida, hepph/0108152; Y. Nomura, hep-ph/0108170; Q. Shafi and Z. Tavartkiladze, hepph/0108247; L. Hall, J. March-Russell, T. Okui and D. Smith, hep-ph/0108161; N. Haba, hep-ph/0110164; R. Barbieri, L. Hall and Y. Nomura, hep-ph/0110102; L. Hall and Y. Nomura, hep-ph/0111068; M. Kubo, C. S. Lim, H. Yamashita, hep-ph/0111327; G. Bhattacharyya and K. Sridhar, hep-ph/0111345; H. D. Kim, J. E. Kim, H. M. Lee, hep-ph/0112094; A. Hebecker, hep-ph/0112230; T. Li and W. Liao, hep-ph/0202090; L. J. Hall and Y. Nomura, hep-ph/0202107; S. Dimopoulos, D. E. Kaplan and N. Weiner, hep-ph/0202136; T. Li and W. Liao, hepth/0207126; Q. Shafi and Z. Tavartkiladze, hep-ph/0210181; J. Jiang, T. Li and W. Liao, hep-ph/0210436; N. Haba and Y. Shimizu, hep-ph/0212166; B. Kyae and Q. Shafi, hep-ph/0212331; H. D. Kim and S. Raby, hep-ph/0212348; N. Haba and Y. Shimizu, hep-ph/0212384; N. Cosme and J. M. Frere, hep-ph/0303037; I. Gogoladze, Y. Mimura and S. Nandi, ep-ph/0304118; C. Biggio, F. Feruglio, I. Masina and M. Perez-Victoria, hep-ph/0305129; A. Hebecker and M. Ratz, hep-ph/0306049; N. Haba, Y. Hosotani and Y. Kawamura, hep-ph/0309088.

[24] T. Li, Eur. Phys. J. C 24, 595 (2002).

[25] C. S. Huang, J. Jiang, T. Li and W. Liao, Phys. Lett. B 530, 218 (2002).

[26] T. Li, Nucl. Phys. B 633, 83 (2002).

[27] T. Watari and T. Yanagida, Phys. Lett. B 532, 252 (2002).

[28] I. Gogoladze, Y. Mimura and S. Nandi, Phys. Lett. B 562, 307 (2003).

[29] S. L. Adler, Phys. Lett. B 533, 121 (2002).

[30] J. Strathdee, Int. J. Mod. Phys. A 2, 273 (1987). 
[31] Y. Imamura, T. Watari and T. Yanagida, Phys. Rev. D 64, 065023 (2001).

[32] N. Marcus, A. Sagnotti and W. Siegel, Nucl. Phys. B 224, 159 (1983).

[33] N. Arkani-Hamed, T. Gregoire and J. Wacker, hep-th/0101233.

[34] R. Slansky, Phys. Rept. 79 (1981) 1.

[35] M. B. Green and J. H. Schwarz, Phys. Lett. B 149, 117 (1984).

[36] M. Dine, N. Seiberg and E. Witten, Nucl. Phys. B 289, 589 (1987).

[37] N. Arkani-Hamed, A. G. Cohen and H. Georgi, hep-th/0103135; C. A. Scrucca, M. Serone, L. Silvestrini and F. Zwirner, hep-th/0110073; L. Pilo and A. Riotto, hep-th/0202144; R. Barbieri, R. Contino, P. Creminelli, R. Rattazzi and C.A. Scrucca, hep-th/0203039; H. D. Kim, J. E. Kim and H. M. Lee, hep-th/0204132; S. Groot Nibbelink, H. P. Nilles and M. Olechowski, hep-th/0205012.

[38] C. G. Callan and J. A. Harvey, Nucl. Phys. B250 (1985) 427; M. B. Green, J. A. Harvey and G. W. Moore, Class. Quant. Grav. 14, 47 (1997).

[39] R. Sekhar Chivukula, D. A. Dicus and H. J. He, Phys. Lett. B 525, 175 (2002); R. S. Chivukula, D. A. Dicus, H. J. He and S. Nandi, Phys. Lett. B 562, 109 (2003); C. Csaki, C. Grojean, H. Murayama, L. Pilo and J. Terning, hep$\mathrm{ph} / 0305237$. 
Table 1: Model I. Under the $U(1)_{X}$ gauge symmetry and $U(1)_{R}^{I} \times U(1)_{F}^{I} R$ symmetry, the quantum numbers for the Standard Model fermions $\mathbf{1 6}_{a^{\prime}}, \mathbf{1 6}_{3}$; Higgs fields $H, \mathbf{4 5}_{H}$, $\psi_{H}, \bar{\psi}_{H}$; vector like particles $\psi, \bar{\psi}, \psi^{a^{\prime}}, \bar{\psi}_{a^{\prime}}, \psi_{a^{\prime}}, \bar{\psi}^{a^{\prime}}$; three $S O(10)$ singlets $N_{a^{\prime}}$ and $N_{3}$; and Higgs fields for flavour symmetry breaking $\phi^{a^{\prime}}, S^{a^{\prime} b^{\prime}}$, and $A^{a^{\prime} b^{\prime}}$. In this Table, $Q_{X}, Q_{R}$ and $Q_{F}$ are free parameters. And the $U(1)_{R}^{I} \times U(1)_{F}^{I}$ charges for $\psi_{H}\left(Q_{\psi_{H}}^{R}\right.$ and $\left.Q_{\psi_{H}}^{F}\right)$ are arbitrary because we have no constraints from superpotential.

\begin{tabular}{|c|c|}
\hline Particles & $U(1)_{X} \times U(1)_{R}^{I} \times U(1)_{F}^{I}$ \\
\hline $\mathbf{1 6} \mathbf{6}_{a^{\prime}}$ & $(-3 ;-1 ;-1 / 2)$ \\
\hline $\mathbf{1 6} \mathbf{6}_{3}$ & $(-3 ;-1 ;+1)$ \\
\hline$H$ & $(+6 ;-1 ;-2)$ \\
\hline $\mathbf{4 5 _ { H }}$ & $\left(-Q_{X} ;-Q_{R} ;-Q_{F}+3\right)$ \\
\hline$\psi_{H}$ & $\left(0 ; Q_{\psi_{H}}^{R} ; Q_{\psi_{H}}^{F}\right)$ \\
\hline $\bar{\psi}_{H}$ & $\left(Q_{X} / 2+3 ; Q_{R} / 2-1 / 2 ; Q_{F} / 2-5 / 2\right)$ \\
\hline$\psi$ & $\left(-Q_{X}-3 ;-Q_{R}-1 ;-Q_{F}+4\right)$ \\
\hline $\bar{\psi}$ & $\left(Q_{X}+3 ; Q_{R}-2 ; Q_{F}-4\right)$ \\
\hline$\psi^{a^{\prime}}$ & $(+3 ;-2 ;-5 / 2)$ \\
\hline $\bar{\psi}_{a^{\prime}}$ & $\left(-Q_{X}-3 ;-Q_{R}-1 ;-Q_{F}+5 / 2\right)$ \\
\hline$\psi_{a^{\prime}}$ & $\left(Q_{X}+3 ; Q_{R}-2 ; Q_{F}-5 / 2\right)$ \\
\hline $\bar{\psi}^{a^{\prime}}$ & $\left(-Q_{X} / 2 ;-Q_{R} / 2-3 / 2 ;-Q_{F} / 2\right)$ \\
\hline$N_{a^{\prime}}$ & $\left(Q_{X} ; Q_{R} ; Q_{F}\right)$ \\
\hline$N_{3}$ & $\left(-Q_{X} / 2 ;-Q_{R} / 2-3 / 2 ;-Q_{F} / 2+3 / 2\right)$ \\
\hline$\phi^{a^{\prime}}$ & $(0 ;+3)$ \\
\hline$S^{a^{\prime} b^{\prime}}$ & \\
\hline$A^{a^{\prime} b^{\prime}}$ & \\
\hline & \\
\hline
\end{tabular}


Table 2: Model II. Under the $U(1)_{X}$ gauge symmetry and $U(1)_{R}^{I I} \times U(1)_{F}^{I I} R$ symmetry, the quantum numbers for the Standard Model fermions $\mathbf{1 6}_{a^{\prime}}, \mathbf{1 6}_{3}$; Higgs fields $H, \mathbf{4 5}_{H}$, $\psi_{H}, \bar{\psi}_{H}$; vector like particles $\Upsilon_{0}, \Upsilon_{0}^{\prime}, \psi, \bar{\psi}, \psi^{a^{\prime}}, \bar{\psi}_{a^{\prime}}, \psi_{a^{\prime}}, \bar{\psi}^{a^{\prime}}$; three $S O(10)$ singlets $N_{a^{\prime}}$ and $N_{3}$; and Higgs fields for flavour symmetry breaking $\phi^{a^{\prime}}, S^{a^{\prime} b^{\prime}}$, and $A^{a^{\prime} b^{\prime}}$. In this Table, $Q_{X}$ and $Q_{F}$ are free parameters. And the $U(1)_{F}^{I I}$ charge for $\psi_{H}\left(Q_{\psi_{H}}^{F}\right)$ is arbitrary because we have no constraints from superpotential.

\begin{tabular}{|c|c|}
\hline Particles & $U(1)_{X} \times U(1)_{R}^{I I} \times U(1)_{F}^{I I}$ \\
\hline $\mathbf{1 6 _ { a ^ { \prime } }}$ & $(-3 ;+1 ; 0)$ \\
\hline $\mathbf{1 6} \mathbf{6}_{3}$ & $(-3 ;+1 ;-1 / 2)$ \\
\hline$H$ & $(+6 ; 0 ; 0)$ \\
\hline $\mathbf{4 5 _ { H }}$ & $\left(-Q_{X} ; 0 ;-Q_{F}-1\right)$ \\
\hline$\psi_{H}$ & $\left(0 ; 0 ; Q_{\psi_{H}}^{F}\right)$ \\
\hline $\bar{\psi}_{H}$ & $\left(Q_{X} / 2+3 ; 0 ; Q_{F} / 2+1 / 2\right)$ \\
\hline$\Upsilon_{0}$ & $(-3 ; 0 ;-1)$ \\
\hline$\Upsilon_{0}^{\prime}$ & $(+3 ; 2 ; 0)$ \\
\hline$\psi$ & $\left(-Q_{X}-3 ;+1 ;-Q_{F}-3 / 2\right)$ \\
\hline $\bar{\psi}$ & $\left(Q_{X}+3 ;+1 ; Q_{F}+1 / 2\right)$ \\
\hline$\psi^{a^{\prime}}$ & $(-3 ;+1 ;-1)$ \\
\hline $\bar{\psi}_{a^{\prime}}$ & $(+3 ;+1 ; 0)$ \\
\hline$\psi_{a^{\prime}}$ & $\left(-Q_{X}-3 ;+1 ;-Q_{F}-1\right)$ \\
\hline $\bar{\psi}^{a^{\prime}}$ & $\left(Q_{X}+3 ;+1 ; Q_{F}\right)$ \\
\hline$N_{a^{\prime}}$ & $\left(-Q_{X} / 2 ;+1 ;-Q_{F} / 2-1 / 2\right)$ \\
\hline$N_{3}$ & $\left(-Q_{X} / 2 ;+1 ;-Q_{F} / 2-1\right)$ \\
\hline$\phi^{a^{\prime}}$ & $\left(Q_{X} ; 0 ; Q_{F}+1 / 2\right)$ \\
\hline$S^{a^{\prime} b^{\prime}}$ & $\left(Q_{X} ; 0 ; Q_{F}\right)$ \\
\hline$A^{a^{\prime} b^{\prime}}$ & $(0 ; 0 ;-1)$ \\
\hline & \\
\hline
\end{tabular}

